\title{
On the regularity of edges in image segmentation*
}

\author{
by
}

\author{
A. BONNET \\ Université de Cergy-Pontoise, \\ 2, avenue Adolphe Chauvin, 95302 Cergy-Pontoise Cedex \\ and \\ École Normale Supérieure, \\ 45, rue d'Ulm, 75005 Paris.
}

ABSTRACT. - We present a series of results on the regularity of edges in the Mumford-Shah minimization process. We prove that an isolated connected component of the edge set is a finite union of $C^{1,1}$ arcs. A fundamental step of the proof is the characterization of the four possible global-minimizers having a connected edge.

RÉsumé. - Nous présentons une série de résultats sur la régularité des contours des minima de la fonctionnelle de Mumford-Shah. Sous certaines hypothèses de connexité on montre que le contours est une union finie d'arcs $C^{1,1}$. Une étape fondamentale de la démonstration est la caractérisation des quatre possibles minima globaux ayant un contour connexe.

\section{CONTENTS}

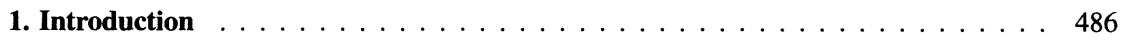

2. Blow-up procedure . . . . . . . . . . . . . . . . . . . . . . . . . 490

2.1. Definition: blow-up, global minimizer . . . . . . . . . . . . . . 490

2.2. Blow-up, limits are global-minimizers . . . . . . . . . . . . . . . . 492

* These results have been announced at the "Geometry driven diffusion in vision" workshop in Stockholm (May. 94) and at the conference "Variational methods for discontinuous structures" in Como (Sept. 94).

Annales de l'Institut Henri Poincaré - Analyse non linéaire - 0294-1449

Vol. 13/96/04/\$ 4.00/

(C) 1996 L'Association Publications de l'Institut Henri Poincaré. Published by Elsevier B.V. All rights reserved 
2.3. Sufficient condition for $K_{0}$ to be connected 496

3. A monotonicity formula . . . . . . . . . . . . . . . . . . . . . 499

3.1. Global-minimizers of $J^{0} \ldots \ldots \ldots \ldots \ldots$. . . . . . . . 499

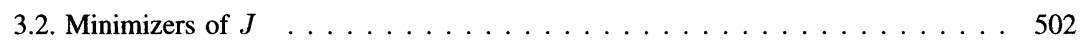

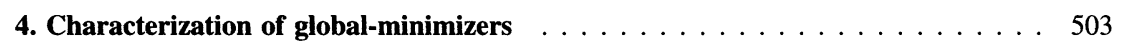

5. Finite number of pieces $\ldots \ldots \ldots \ldots \ldots \ldots$

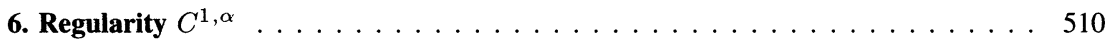

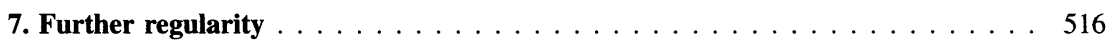

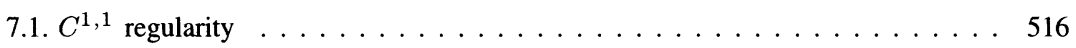

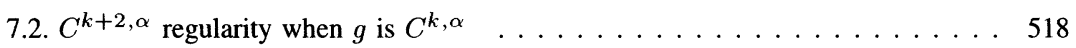

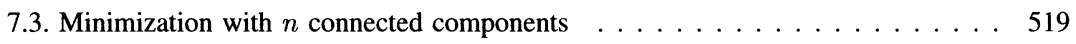

8. Regularity almost everywhere $\ldots \ldots \ldots \ldots \ldots 20 \ldots \ldots \ldots$

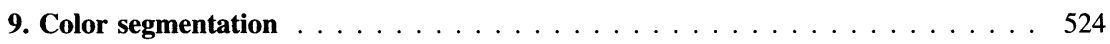

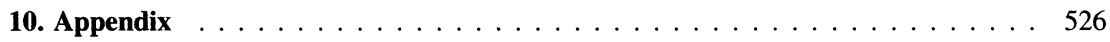

\section{INTRODUCTION}

In computer vision, an image is characterized by the collection of numbers giving the grey-level at each point of the screen. Mathematically, we will say that an image is given by a real $L^{\infty}$ function $g$ (the grey level) defined on a domain $\Omega$ (the screen) of $\mathbf{R}^{2}$. At the end of this article we will consider color images. In this case, $g$ will be a vector function (of the red, blue and green intensity) and will take its values in a cube of $\mathbf{R}^{3}$.

In most images, human vision is able to detect structures, shapes of objects, etc. Those structures appear, when, for instance, the image is made of patches in which the grey level has but little variations. Sharp discontinuities are visible at the boundary of these patches. They are introduced by edges of objects, shadows, overlapping objects, etc.

The object of image segmentation is to extract, by a systematic algorithm, the meaningful discontinuities. The result of this operation will be a piecewise regular image $u$ approximating the true image $g$ and a set $K$ of discontinuities of $u$. The set $K$ is an estimation of the contours of the image. Although there is a number of different algorithms and softwares for image segmentation, it appears that all rely on the same principle [17]: 
the minimization of an energy. Moreover, this energy is essentially made of the three terms of the Mumford-Shah functional.

In [19], Mumford and Shah proposed to define $(u, K)$, where $u \in$ $C^{1}(\Omega \backslash K)$ and $K$ is a closed set, as minimizers of the functional:

$$
J(u, K)=\int_{\Omega \backslash K}\|\nabla u\|^{2}+\mu \int_{\Omega}(u-g)^{2}+\nu \mathcal{H}^{1}(K)
$$

The first term of this energy penalizes the variations of $u$ outside the edge set $K$, the second term is the distance to the true image and the third term is the 1-dimensional Hausdorff measure of the edge set $K$ ([17] and [14]). In the energy $J, \mu$ and $\nu$ denote two positive constants. By a normalization we may assume without loss of generality that $\mu=\nu=1$. For a simple geometric image one would expect that the set $K$ is regular. However, for pictures of the real world it is not obvious that the edge set should be simple. More precisely, one cannot eliminate a priori the possibility of having a tree like edge set (Fig. 1) or lots of very small pieces (Fig. 2) or multibranch stars (Fig. 3). Mumford and Shah conjectured that there exists a minimizer of $J$ such that the edge set $K$ is the union of a finite set of $C^{1}$-arcs and that each arc may end either as a crack-tip or in a triple junction (Fig. 4).

Existence of minimizers in the class of $S B V$ functionals has been proved in [2], [11] and [6]. A function $u$ is in $B V(\Omega)$ if the distributional gradient $D u$ is a bounded Radon measure on $\Omega$ with values in $\mathbf{R}^{2}$. If $S_{u}$ denotes the singular set of $u \in B V(\Omega)$ then $\mathcal{H}^{1}\left(S_{u}\right)<\infty$ and for $\mathcal{H}^{1}$-a.e. $x \in S_{u}$ one can define a normal vector $\nu_{u}$ and limit values $u^{+}$and $u^{-}$of $u$ on both sides of $S_{u}$. A function $u$ is in $S B V(\Omega)$ (Special function of Bounded Variations) if $u \in B V(\Omega)$ and if its distributional gradient $D u$ satisfies, for

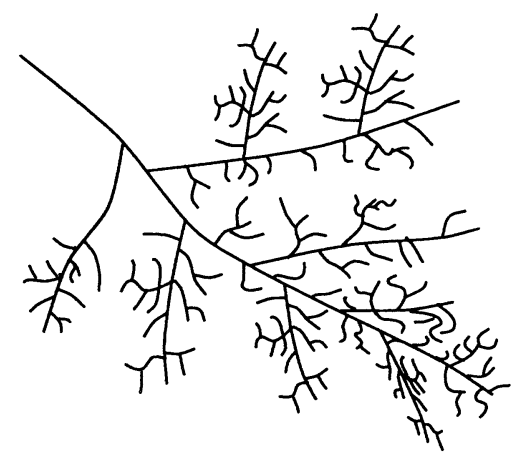

Fig. 1. 
A. BONNET

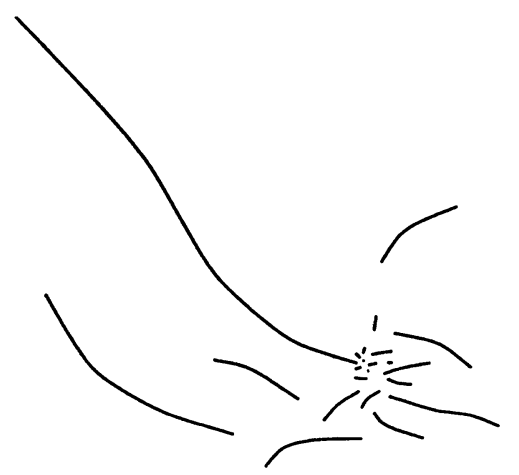

Fig. 2.

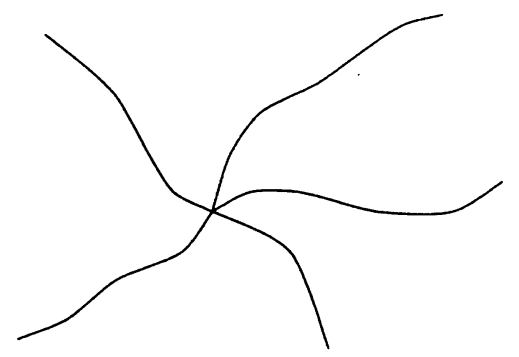

Fig. 3.
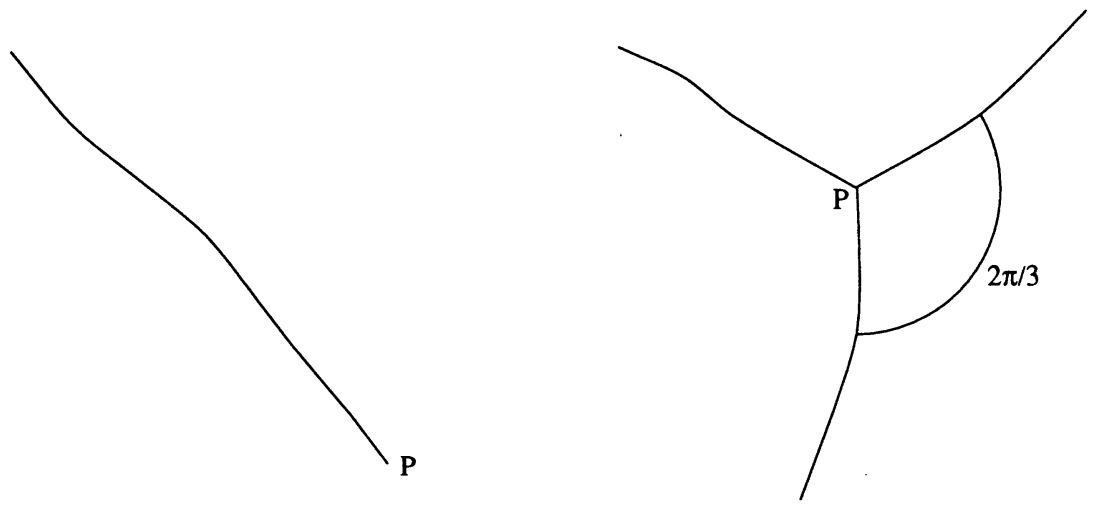

Crack-tip

Fig. 4. 
every $C_{0}^{1}$ vector field $\phi: \Omega \rightarrow \mathbf{R}^{2}$ :

$$
\int_{\Omega} \phi . D u=\int_{\Omega} \phi \cdot \nabla u+\int_{S_{u}}\left(u^{+}-u^{-}\right) \phi \cdot \nu_{u} d \mathcal{H}^{1}
$$

where $\nabla u$ is in $L^{2}\left(\Omega, \mathbf{R}^{2}\right)$.

It is proved in [6] that there is a correspondence between $S B V$ minimizers and pairs $(u, K)$ minimizing $J$. In the following, we choose to work with the initial formula of the function as depending upon $(u, K)$, $u \in C^{1}(\Omega \backslash K) \cap W^{1,2}(\Omega \backslash K)$ and $K$ is a Hausdorff 1-dimensional closed set with finite Hausdorff measure.

Notice that we do not change $J(u, K)$ by adding a set of zero $\mathcal{H}^{1}$ measure to $K$. Therefore, we will assume in the following that $K$ is a minimal closed set: there is no closed set $\tilde{K} \subset K, \tilde{K} \neq K$, such that $u$ can be extended on $\Omega \backslash \tilde{K}$ with $J(u, \tilde{K}) \leq J(u, K)$. Under this assumption, $K$ is an Ahlfors regular set: there are two positive constants $c, C$ such that [6]:

$$
\text { if } P \in K \quad \text { then } \forall r>0, \quad c r \leq \mathcal{H}^{1}\left(K \cap B_{r}(P)\right) \leq C r \text {. }
$$

In [7], it is proved that $K$ is contained in a single rectifiable Ahlfors regular curve whose length is proportional (with a universal ratio) to the length of $K$.

In this paper, we are interested in the local regularity of the edge set (see also [8] and [3]-[4]) and in the number of arcs. Our main ingredient is the characterization of all global-minimizers (i.e. pairs $(u, K)$ defined on $\Omega=\mathbf{R}^{2}$ and such that for all balls $B_{R}$ the energy $J_{B_{R}}^{0}(u, K)=\int_{B_{R} \backslash K}\|\nabla u\|^{2}+\mathcal{H}^{1}\left(K \cap B_{R}\right)$ cannot be reduced by a compact perturbation of $u$ and $K$ inside the ball $B_{R}$.) Under the assumption that $K$ is connected, it will be proved that there are only four types of globalminimizers. The characterization is carried out by a monotonicity property on the function $r \mapsto \frac{\int_{B_{r} \backslash K}\|\nabla u\|^{2}}{r}$. We will be able to prove that every blow-up limit is a global-minimizer. The characterization of all globalminimizers and therefore of the blow-up limits give strong information on the local behavior of $K$ and $u$.

THEOREM 1.1. - If $G$ is an isolated connected component of $K$ then, it is the union of a finite set of $C^{1}$ arcs. These arcs are $C^{1,1}$ away from crack-tips and can only merge through triple junctions.

More precisely,

THEOREM 1.2. - Let $P$ be a point of the edge set $K$. If $B_{R}(P)$ intersects a single connected component of $K$, then there is a finite set of arcs $l_{j}$, 
$j=1, . ., k$, with the same regularity property as above and such that

$$
K \cap B_{R / 2}(P)=\left(\cup_{j=1}^{k} l_{j}\right) \cap B_{R / 2}(P) .
$$

Notice that this does not allow a minimizer to have an infinite number of arbitrary small pieces connected to each other. In July 1994, G. David announced a $\mathcal{H}^{1}$-almost-everywhere regularity result, [8]. Actually, the method used to prove the theorems above allows one to get

THEOREM 1.3. - For $\mathcal{H}^{1}$ almost every $P$ in $K$ there is a neighborhood $B_{r}(P)$ of $P$ in which $K$ is a $C^{1,1}$ arc.

The above results were derived when $g$ is $L^{\infty}$. If one assumes more regularity on $g$ one may expect more regularity on $K$.

THEOREM 1.4. - Assume that $g$ is $C^{k, \alpha}$. If, in an open neighborhood $U$ of $P \in K, K \cap U$ is a $C^{1}$ arc then it is $C^{k+2, \alpha}$ in $U$.

Lastly, we would like to point out that it can be interesting to look for a minimizer $\left(u^{n}, K^{n}\right)$ of $J$ that has at most $n$ connected components (see [17]). We have the complete result:

THEOREM 1.5. - The edge set $K^{n}$ is the union of a finite set of $C^{1}$ arcs, $C^{1,1}$ away from the crack-tips, and merging through triple junctions.

To conclude this paper, we show, in section 9, that the analysis above holds for a model of color segmentation. A point of the image is characterized by its Red, Green and Blue intensity. In this case, $u(x)$ is a vector of $[0,1]^{3}$ with a specific Riemannian metric which gives a slightly different energy functional [10].

Open questions. It is not known whether the edge set is regular up to the crack-tip.

The finite number of arcs is still an open question. Notice that if the characterization of global-minimizers was achieved without the assumption that $K$ is connected, then the conjecture would be solved.

\section{BLOW-UP PROCEDURE}

\subsection{Definition: blow-up, global minimizer}

For a given open set $\omega$ and a piecewise continuous function $\psi$ defined on $\partial \omega$ we may study the minimization of the functional

$$
J_{\omega}(v, \tilde{K})=\int_{\omega \backslash \tilde{K}}\|\nabla v\|^{2}+\int_{\omega}(v-g)^{2}+\mathcal{H}^{1}(\tilde{K} \cap \omega)
$$

under the condition $v=\psi$ on $\partial \omega$. 
Assume, as in the previous section, that $(u, K)$ denotes a minimum segmentation. If $\omega \subset \Omega$ is such that $\partial \omega \cap K$ is finite, we define the trace $\psi$ of $u$ on $\partial \omega$. It is straightforward to see that $(u, K)$ minimizes the functional $J_{\omega}$ with $\psi$ as Dirichlet condition. This means that one cannot modify $u$ and $K$ inside $\omega$ and reduce the total energy $J=J_{\omega}+J_{\Omega \backslash \omega}$.

In the study of free-boundary problems, it is common to use blow-up procedures ([1], [16], etc.). Here, we introduce, for a given origin, the blow-up sequences $u_{\varepsilon}, g_{\varepsilon}, K_{\varepsilon}$ :

$$
\begin{aligned}
& u_{\varepsilon}(X)=\frac{u(\varepsilon X)-c_{\varepsilon}(X)}{\sqrt{\varepsilon}}, \\
& g_{\varepsilon}(X)=\frac{g(\varepsilon X)-c_{\varepsilon}(X)}{\sqrt{\varepsilon}}, \\
& K_{\varepsilon}=\left\{X \in \mathbf{R}^{2}, \varepsilon X \in K\right\} .
\end{aligned}
$$

The real function $c_{\varepsilon}(X)$ is piecewise constant. It is introduced in order to be able to pass to the limit $\varepsilon \rightarrow 0$ in such a way that $u_{\varepsilon}$ converges almost everywhere to some finite value. This will be detailed in the second part of this section. We first take $c \equiv 0$ and introduce:

$$
\begin{aligned}
U_{\varepsilon}(X) & =\frac{u(\varepsilon X)}{\sqrt{\varepsilon}}, \\
G_{\varepsilon}(X) & =\frac{g(\varepsilon X)}{\sqrt{\varepsilon}} .
\end{aligned}
$$

We notice that $\nabla U_{\varepsilon}(X)=\sqrt{\varepsilon} \nabla u(\varepsilon X)$ and that a ball $B_{\varepsilon}$ with radius $\varepsilon$ becomes $B_{1}$ by the blow-up procedure. Thus

$$
J_{B_{\varepsilon}}(u, K)=\varepsilon \int_{B_{1} \backslash K_{\varepsilon}}\left\|\nabla U_{\varepsilon}\right\|^{2}+\varepsilon^{3} \int_{B_{1}}\left(U_{\varepsilon}-G_{\varepsilon}\right)^{2}+\varepsilon \mathcal{H}^{1}\left(K_{\varepsilon} \cap B_{1}\right) .
$$

We introduce the new functionals $J_{\omega}^{\varepsilon}$ :

$$
J_{\omega}^{\varepsilon}\left(U_{\varepsilon}, K_{\varepsilon}\right)=\int_{\omega \backslash K_{\varepsilon}}\left\|\nabla U_{\varepsilon}\right\|^{2}+\varepsilon^{2} \int_{\omega}\left(U_{\varepsilon}-G_{\varepsilon}\right)^{2}+\mathcal{H}^{1}\left(K_{\varepsilon} \cap \omega\right) .
$$

With this definition we have the straightforward statement on the minimization under Dirichlet conditions on $\partial B_{\varepsilon}$ and $\partial B_{1}$ respectively:

$$
(u, K) \text { minimizes } J_{B_{\varepsilon}} \Leftrightarrow\left(U_{\varepsilon}, K_{\varepsilon}\right) \text { minimizes } J_{B_{1}}^{\varepsilon} \text {. }
$$

We remark that in the definition of $J_{\omega}^{\varepsilon}$, the term $\varepsilon^{2} \int_{\omega}\left(U_{\varepsilon}-G_{\varepsilon}\right)^{2}$ is of order $\varepsilon$. In the limit $\varepsilon=0$ we define

$$
J_{\omega}^{0}\left(u_{0}, K_{0}\right)=\int_{\omega \backslash K_{0}}\left\|\nabla u_{0}\right\|^{2}+\mathcal{H}^{1}\left(K_{0} \cap \omega\right) .
$$


In the following we will be interested in minimizers of the functional $J^{0}$ that are defined on the whole plane. Since there is no more image $g$ in the functional $J^{0}$ and since the domain is now unbounded, we introduce a notion of compact-perturbation-global-minimizer of $J^{0}$. For simplicity, this will be referred to in the following as "global-minimizer of $J^{0}$ ".

DEFinition 2.1. - A segmentation $(u, K)$ is a global-minimizer of $J^{0}$ if it is defined on the whole space and if for any bounded open sub-domains $U, V$ of $\mathbf{R}^{2}$ with $U \subset \subset V$ there are no function $\tilde{u}$ and closed edge set $\tilde{K}$ defined on $V$ such that:

(i) $\tilde{u}$ and $u$ (resp. $\tilde{K}$ and $K$ ) coincide in $V \backslash \bar{U}$,

(ii) for every pair of points $X, Y$ in $V \backslash(\bar{U} \cup K)$, if $X$ and $Y$ are in two distinct connected components of $V \backslash K$ so they are in $V \backslash \tilde{K}$,

(iii) $J_{V}^{0}(\tilde{u}, \tilde{K})<J_{V}^{0}(u, K)$.

Notice that we take $V \neq U$ in order to avoid problems arising from $K \cap \partial U \neq \emptyset$. Condition (ii) is coherent with the introduction of the piecewise constant function $c_{\varepsilon}(X)$ in the definition of the blow-up sequences. Indeed, if $P$ is a regular point of $K$, then the blow-up limit at $P,\left(u_{0}, K_{0}\right)$, is the segmentation of the plane such that $K_{0}$ is a straight line $\{y=0\}$ and $u$ is constant on both sides of $K\left(u_{0}(x, y)=c^{+}\right.$if $y>0, u_{0}(x, y)=c^{-}$ if $y<0)$. With condition (ii), $\left(u_{0}, K_{0}\right)$ is a global-minimizer. However, whatever the value of $c^{+}$and $c^{-}$, the value of $J_{B_{2 R}}^{0}$ can be reduced for large enough $R$ by excising a large segment out of $K$ (this excision violates (ii)).

\subsection{Blow-up limits are global-minimizers}

The fundamental result in the blow-up procedure is the following theorem which says that blow-up sequences converge to global-minimizers.

THeOREM 2.2. - Let $(u, K)$ be a minimal segmentation of $J$. The blowup sequence $\left(u_{\varepsilon}, K_{\varepsilon}\right)$ is such that there is a subsequence converging to $\left(u_{0}, K_{0}\right)$ in the following sense:

$K_{\varepsilon_{q}} \rightarrow K_{0}$ locally in the Hausdorff metric,

$u_{\varepsilon_{q}} \rightarrow u_{0}$ strongly in $H_{l o c}^{1}\left(\mathbf{R}^{2} \backslash K_{0}\right)$,

$J_{\omega}^{0}\left(u_{0}, K_{0}\right) \leq \liminf J_{\omega}^{\varepsilon_{q}}\left(U_{\varepsilon_{q}}, K_{\varepsilon_{q}}\right)$ for any bounded open set $\omega$,

$J_{B_{r}}^{0}\left(u_{0}, K_{0}\right)=\lim J_{B_{r}}^{\varepsilon_{q}}\left(U_{\varepsilon_{q}}, K_{\varepsilon_{q}}\right)$ for almost every $r>0$.

Moreover $\left(u_{0}, K_{0}\right)$ is a global-minimizer.

THEOREM 2.3. - If $\left(v_{n}, K_{n}\right)$ minimizes $J$ for an image $g^{n}$ such that $\left\|g^{n}\right\|_{L^{\infty}} \leq 1$ (or other uniform $L^{p}$ bound with $p \geq 2$ ) the blow-up sequence 
$\left(v_{\varepsilon_{n}}^{n}, K_{\varepsilon_{n}}^{n}\right)$ with $\varepsilon_{n} \rightarrow 0$ has a convergent subsequence to a global-minimizer $\left(v_{0}, K_{0}\right)$ of $J^{0}$ in the same sense as in theorem 2.2.

Remark 2.4. - Given a blow-up sequence $\left(u_{\varepsilon}, K_{\varepsilon}\right)$ there may exist several blow-up limits. The object of section 4 will be to characterize all the possible blow-up limits.

Proof. - Theorem 2.3 is a straightforward generalization of theorem 2.2. Therefore we will concentrate on the proof of theorem 2.2. The proof of convergence relies as in [6] and [11] on the $L^{2}$ bound on $\nabla u$ and on the compactness of Hausdorff metric. However, because of the introduction of the functions $c_{\varepsilon}$ in the definition of $u_{\varepsilon}$ and since $u_{\varepsilon}$ is not obviously bounded, we will detail the proof hereafter.

(i) Convergence of $K_{\varepsilon}$ to $K_{0}$

First we recall the definition of the Hausdorff distance $\mathbf{d}$ of two sets $A$ and $B$ (for two points $x$ and $y, d(x, y)$ denotes the usual euclidean distance):

$$
\begin{aligned}
\mathbf{d}(A, B) & =\max \left(\sup _{x \in A} d(x, B), \sup _{y \in B} d(y, A)\right) \\
d(x, B) & =\inf _{y \in B} d(x, y) .
\end{aligned}
$$

The compactness of the Hausdorff metric $\mathbf{d}$ for subsets of a compact domain gives the existence of a subsequence $K_{\varepsilon_{q}}$ converging locally to a set $K_{0}$.

(ii) Convergence of $u_{\varepsilon}$ to $u_{0}$

In each connected component of $\mathbf{R}^{2} \backslash K_{0}$ we choose a point $Y_{i}$. Let $c_{\varepsilon}$ be the piecewise constant function defined on $\mathbf{R}^{2} \backslash K_{0}$ by

$$
\begin{aligned}
c_{\varepsilon}(X)= & u_{\varepsilon}\left(Y_{i}\right) \text { if } X \text { and } Y_{i} \text { are in the same } \\
& \text { connected component of } \mathbf{R}^{2} \backslash K_{0} .
\end{aligned}
$$

We study now the convergence of $u_{\varepsilon}$. We notice that the gradients $\nabla U_{\varepsilon}$ are uniformly locally bounded in the $L^{2}$ norm (by an excision argument one gets $J_{B_{R}}^{\varepsilon}\left(U_{\varepsilon}, K_{\varepsilon}\right) \leq 2 \pi R$, see [6] for the details). Consequently $\int_{B_{R} \backslash\left(K_{0} \cup K_{\varepsilon}\right)}\left\|\nabla u_{\varepsilon}\right\|^{2} \leq 2 \pi R$. Let $F$ be a bounded open set, $F \subset \subset \mathbf{R}^{2} \backslash K_{0}$. For $\varepsilon$ small, $F \subset \Omega_{\varepsilon} \backslash K_{\varepsilon}$. Since $\left|\Delta u_{\varepsilon}\right|=\varepsilon^{2}\left|U_{\varepsilon}-G_{\varepsilon}\right| \leq \varepsilon^{3 / 2}, \Delta u_{\varepsilon}$ is Vol. 13, $\mathrm{n}^{\circ}$ 4-1996. 
uniformly bounded for $\varepsilon \leq 1$ in $F$. The $L^{2}$ bound on $\nabla u_{\varepsilon}$ and the $L^{\infty}$ bound on $\Delta u_{\varepsilon}$ give a bound on $\left\|\nabla u_{\varepsilon}\right\|_{L^{p}(F)}$, for all $p \geq 1$, uniform in $\varepsilon$ small. By Sobolev embedding, we get a uniform bound on $\left\|u_{\varepsilon}\right\|_{C^{0, \alpha}(F)}$. Consequently, $\exists M, \forall \varepsilon<\varepsilon_{0}$

$$
\left\|u_{\varepsilon}\right\|_{W^{2, p}(F)}<M
$$

and a subsequence of $u_{\varepsilon}$ converges to $u_{0}$ in $W_{l o c}^{1, p}\left(\mathbf{R}^{2} \backslash K_{0}\right)$.

\section{(iii) The blow-up limit $\left(u_{0}, K_{0}\right)$ is a global-minimizer}

Let us finally check that $\left(u_{0}, K_{0}\right)$ is a global-minimizer. We notice that by construction,

$$
\int_{B_{R} \backslash K_{0}}\left\|\nabla u_{0}\right\|^{2} \leq \liminf \int_{B_{R} \backslash K_{\varepsilon}}\left\|\nabla u_{\varepsilon}\right\|^{2} .
$$

Inequality (2.12) and a lower semicontinuity lemma for the Hausdorff measure [6] are the two main ingredients of the proof of the claim that $\left(u_{0}, K_{0}\right)$ is a global-minimizer.

We recall definition 0.9 , lemma 0.10 and theorem 3.38 of [6]:

Definition 2.5. - [6] Let A be a Borel subset of $\bar{\Omega}$. We say that $A$ satisfies the concentration property in $\Omega$ if for every $\varepsilon>0$ there exists $\alpha=\alpha(\varepsilon)>0$ such that, if $B_{R}=B\left(x_{0}, R\right)$ is any disk contained in $\Omega$ with $x_{0} \in A$ and $0<R<1$, then there exists a disk $B(x, r)$ contained in $B_{R}$ such that

$$
\begin{aligned}
& \operatorname{diam}(B(x, r)) \geq \alpha \operatorname{diam}\left(B_{R}\right) \\
& \mathcal{H}^{1}(B(x, r) \cap A) \geq(1-\varepsilon) \operatorname{diam}(B(x, r)) .
\end{aligned}
$$

LEMMA 2.6. - [6] Let $\left(K_{k}\right)$ be a sequence of closed subsets of $\Omega$ which converges in the Hausdorff metric to a closed subset $K$ of $\bar{\Omega}$. Assume that the sets $K_{k}$ satisfy the concentration property (definition 2.5) in $\Omega$ uniformly with respect to $k$ (i.e. with $\alpha(\varepsilon)$ independent of $k$ ). Then

$$
\mathcal{H}^{1}(K \cap \Omega) \leq \liminf \mathcal{H}^{1}\left(K_{k} \cap \Omega\right)
$$

THEOREM 2.7. - [6] Let $u, K$ be a minimum segmentation of J. Then $K$ satisfies the concentration property in $\Omega$ with a function $\varepsilon \mapsto \alpha(\varepsilon)$ which does not depend on the data $\Omega, g, u\left(\|g\|_{L^{\infty}} \leq 1\right)$. 
It is straightforward to see that if $K$ satisfies the concentration property, then the blow-up $K_{\varepsilon}$ for $\varepsilon \leq 1$ satisfies the same property with the same function $\varepsilon \mapsto \alpha(\varepsilon)$. Consequently, for the converging subsequence $K_{\varepsilon_{q}}$ of the blow-up procedure we have the following lower semicontinuity property on any open domain $\omega$ of $\mathbf{R}^{2}$,

$$
\mathcal{H}^{1}\left(K_{0} \cap \omega\right) \leq \liminf \mathcal{H}^{1}\left(K_{\varepsilon_{q}} \cap \omega\right)
$$

This, together with (2.12), gives the lower semicontinuity result on the blow-up sequence,

$$
J_{\omega}^{0}\left(u_{0}, K_{0}\right) \leq \liminf J_{\omega}^{\varepsilon_{q}}\left(U_{\varepsilon_{q}}, K_{\varepsilon_{q}}\right) .
$$

We assume by contradiction that $\left(u_{0}, K_{0}\right)$ is not a global-minimizer of $J^{0}$. Then there is $U=B_{R}$ and $V=B_{2 R}$ and a couple $\left(\tilde{u}_{0}, \tilde{K}_{0}\right)$ satisfying conditions (i), (ii) and (iii) of definition 2.1. We construct hereafter a compact perturbation of $\left(U_{\varepsilon_{q}}, K_{\varepsilon_{q}}\right)$ and prove that for large $q,\left(U_{\varepsilon_{q}}, K_{\varepsilon_{q}}\right)$ cannot be a minimizer of $J^{\varepsilon_{q}}$.

Since $\mathcal{H}^{1}\left(K_{0} \cap B_{r}\right) \leq 2 \pi r$, the intersection $K_{0} \cap \partial B_{r}$ is a finite set of points for almost every $r$. We choose $r_{0}, r_{0}>R$ such that $K_{0} \cap \partial B_{r_{0}}$ is finite. We denote $K_{0} \cap \partial B_{r_{0}}=\left\{y_{1}, . ., y_{n}\right\}$. Choose $\zeta>0$ such that

$$
3 n \zeta<J_{B_{2 r_{0}}}^{0}\left(u_{0}, K_{0}\right)-J_{B_{2 r_{0}}}^{0}\left(\tilde{u}_{0}, \tilde{K}_{0}\right) .
$$

We introduce two subsets of $\partial B_{r_{0}}$ :

$$
\begin{aligned}
& L=\partial B_{r_{0}} \cap\left(\cup_{i=1}^{n} B\left(y_{i}, \zeta\right)\right), \\
& \Gamma=\partial B_{r_{0}} \backslash\left(\cup_{i=1}^{n} B\left(y_{i}, \zeta\right)\right) .
\end{aligned}
$$

The set $\Gamma$ is compact in $\mathbf{R}^{2} \backslash K_{0}$. For a subset $A$ of $\mathbf{R}^{2}$ we define $A^{\eta}=\left\{X \in \mathbf{R}^{2}, d(X, A) \leq \eta\right\}$. There is a $\eta>0$ such that $\Gamma^{3 \eta} \subset \mathbf{R}^{2} \backslash K_{0}$. Then for $q$ large, the convergence of $K_{\varepsilon_{q}}$ to $K_{0}$ in the Hausdorff metric gives $\Gamma^{2 \eta} \subset \mathbf{R}^{2} \backslash K_{\varepsilon_{q}}$. Then for $p>2$, the estimates (2.11) and the compact embedding of $W^{2, p}$ in $C^{1, \alpha}\left(\alpha=1-\frac{2}{p}\right)$ gives the convergence of a subsequence $u_{\varepsilon_{q}}$ to $u_{0}$ in $\Gamma_{\eta}$ for the $C^{1, \alpha}$ norm. We introduce the continuous function $w_{q}$ in $\partial B_{r_{0}}$ such that: $w_{q}=u_{\varepsilon_{q}}-u_{0}$ on $\Gamma$ and $w_{q}$ is linear in the polar angle $\theta$ on every connected component of $\partial B_{r_{0}} \backslash \Gamma$ (i.e. $w_{q}\left(r_{0}, \theta\right)=\alpha+\beta \theta$ where $\alpha$ and $\beta$ are chosen such that $w_{q}$ is continuous on $\left.\partial B_{r_{0}}\right)$. Then, for $\|x\|<r_{0}$ we define

$$
w_{q}(x)=\psi\left(\frac{\|x\|}{r_{0}}\right) w_{q}\left(r_{0} \frac{x}{\|x\|}\right)
$$

Vol. 13, $\mathrm{n}^{\circ} 4-1996$. 
where $\psi$ is a $C^{\infty}$ nonnegative function in $[0,1]$ such that $\psi=1$ in a neighborhood of 1 and $\psi=1$ in a neighborhood of 0 . With this construction $w_{q} \in W^{1, \infty}\left(B_{r_{0}}\right)$ and there is a constant $C=C\left(r_{0}, K_{0}, \eta\right)$ such that almost everywhere:

$$
\begin{aligned}
\left\|\nabla w_{q}\right\| & \leq C\left(\left\|\frac{\partial}{\partial \tau}\left(u_{\varepsilon}-u_{0}\right)\right\|_{L^{\infty}(\Gamma)}+\left\|u_{\varepsilon}-u_{0}\right\|_{L^{\infty}(\Gamma)}\right) \\
& \leq C\left\|u_{\varepsilon}-u_{0}\right\|_{C^{1, \alpha}(\Gamma)} .
\end{aligned}
$$

We define $\tilde{U}_{\varepsilon_{q}}$ by

$$
\begin{aligned}
& \tilde{U}_{\varepsilon_{q}}=\tilde{u}_{0}+w_{q}+c_{\varepsilon} \text { in } B_{r_{0}} \backslash K_{0}, \\
& \tilde{U}_{\varepsilon_{q}}=U_{\varepsilon_{q}} \quad \text { in } \mathbf{R}^{2} \backslash\left(B_{r_{0}} \cup K_{0}\right) .
\end{aligned}
$$

and construct $\tilde{K}_{\varepsilon_{q}}$ from $K_{\varepsilon_{q}}$ by adding the piece $L$ and replacing $K_{\varepsilon_{q}}$ by $\tilde{K}_{0}$ inside the ball $B_{r_{0}}$ :

$$
\tilde{K}_{\varepsilon_{q}}=\left(K_{\varepsilon_{q}} \backslash \bar{B}_{r_{0}}\right) \cup L \cup\left(\tilde{K}_{0} \cap B_{r_{0}}\right) .
$$

Then $\tilde{U}_{\varepsilon_{q}}$ is such that

$$
\int_{B_{2 r_{0} \backslash \tilde{K}_{\varepsilon_{q}}}}\left\|\nabla \tilde{U}_{\varepsilon_{q}}\right\|^{2} \rightarrow \int_{B_{2 r_{0}} \backslash \tilde{K}_{0}}\left\|\nabla \tilde{u}_{0}\right\|^{2} .
$$

This, together with (2.16) and (2.14), gives for $q$ large and $\zeta$ small:

$$
J_{B_{2 r_{0}}}^{\varepsilon_{q}}\left(\tilde{U}_{\varepsilon_{q}}, \tilde{K}_{\varepsilon_{q}}\right)<J_{B_{2 r_{0}}}^{\varepsilon_{q}}\left(U_{\varepsilon_{q}}, K_{\varepsilon_{q}}\right)
$$

which contradicts the minimality of $U_{\varepsilon}$.

As a consequence of this argument we have proved that for almost every $r$

$$
J_{B_{r}}^{0}\left(u_{0}, K_{0}\right)=\lim J_{B_{r}}^{\varepsilon_{q}}\left(U_{\varepsilon_{q}}, K_{\varepsilon_{q}}\right)
$$

which completes the proof of theorem 2.2.

\subsection{Sufficient condition for $K_{0}$ to be connected}

In section 4 we will be interested in global-minimizers such that the edge set $K$ is connected. The following lemma will ensure that the edge set $K_{0}$ of the blow-up limit is connected.

LeMmA 2.8. - Assume that $\omega \subset \Omega$ intersects only one connected component of $K$. Let $P_{n}$ be a sequence of points of an open set $U \subset \subset \omega$. For 
every blow-up sequence $u_{n}(X)=\frac{u\left(P_{n}+\varepsilon_{n} X\right)-c_{n}(X)}{\sqrt{\varepsilon_{n}}}, K_{n}=\left\{X, P_{n}+\varepsilon_{n} X \in\right.$ $K\}$ converging to a blow-up limit $\left(u_{0}, K_{0}\right)$, the limit edge set $K_{0}$ is connected.

Proof. - The proof will proceed by contradiction. We assume that $K_{0}$ is not connected. Notice that since $K_{n}$ is a 1-dimensional Hausdorff connected set, it is arc-wise connected: for any two points $X$ and $Y$ in $K_{n}$ there is a continuous function $\psi:[0,1] \rightarrow K_{n}$ such that $\psi(0)=X$ and $\psi(1)=Y$ (see [13]).

The edge set $K_{0}$ is the limit in the Hausdorff distance of the connected edge sets $K_{n}$. Therefore, $K_{0}$ cannot have a bounded isolated connected component and has at least two unbounded connected components. One may choose a point $M$ in one component and a point $N$ in an other. Since $K_{n}$ converges to $K_{0}$ in the Hausdorff distance there are two sequences of points $M_{n} \in K_{n}$ and $N_{n} \in K_{n}$ converging to $M$ and $N$ respectively.

Notation. - Let $A, B$ be two points of $K$. We denote by $d_{K}(A, B)$ the minimal length of an arc connecting $A$ to $B$ in $K .\left(d_{K}(A, B)=+\infty\right.$ if there is no arc connecting $A$ to $B$ in $K$ ).

Since $K_{n}$ is closed there is an arc of minimum length $\psi_{n}$, parameterized by arc-length, connecting the points $M_{n}$ and $N_{n}$ in $K_{n}$ :

$$
\begin{aligned}
& \psi_{n}:\left[0, l_{n}\right] \rightarrow K_{n}, \quad \psi_{n}(0)=M_{n}, \quad \psi_{n}\left(l_{n}\right)=N_{n} \\
& l_{n}=d_{K_{n}}\left(M_{n}, N_{n}\right) .
\end{aligned}
$$

Obviously, this is a Jordan arc ( $\psi_{n}$ is one-to-one) and $\psi_{n}$ is 1-Lipschitz continuous. We notice that $l_{n} \rightarrow+\infty$. Indeed, if $l_{n}$ does not go to $+\infty$ then a subsequence of $\psi_{n}$ will converge to a $\psi$ connecting $M$ to $N$ in $K_{0}$. This contradicts the choice of $M$ and $N$ in two different connected components of $K_{0}$. We choose a new blow-up sequence such that the image by this blow-up of the arc connecting $M_{n}$ to $N_{n}$ is with length 1:

$$
v_{n}(X)=\frac{u\left(P_{n}+\varepsilon_{n} l_{n} X\right)}{\sqrt{\varepsilon_{n} l_{n}}}, \quad G_{n}=\left\{X \in \mathbf{R}^{2}, P_{n}+\varepsilon_{n} l_{n} X \in K\right\} .
$$

To have a blow-up sequence, we have to check that $\varepsilon_{n} l_{n}$ goes to 0 . We argue by contradiction and assume that $\varepsilon_{n} l_{n} \rightarrow k>0$. We introduce

$$
\xi_{n}:[0,1] \rightarrow \mathbf{R}^{2}, \quad \xi_{n}(t)=\varepsilon_{n} \psi_{n}\left(l_{n} t\right)+P_{n}
$$

We notice that, for all $t \in[0,1], \psi_{n}\left(l_{n} t\right) \in K_{n}=\left\{X \in \mathbf{R}^{2}, P_{n}+\varepsilon_{n} X \in\right.$ $K\}$. This implies: $\xi_{n}(t) \in K$. Moreover, since $\varepsilon_{n} l_{n} \rightarrow k, \xi_{n}$ is $2 \mathrm{k}$-Lipschitz Vol. 13, $\mathrm{n}^{\circ} 4-1996$. 
for $n$ large and a subsequence converges uniformly to $\xi:[0,1] \rightarrow K$. We may assume that $P_{n} \rightarrow P \in \omega$. Since $\xi_{n}$ connects the points $\hat{M}_{n}=\varepsilon_{n} M_{n}+P_{n}$ and $\hat{N}_{n}=\varepsilon_{n} N_{n}+P_{n}$ in $K$ we deduce that $\xi(0)=\xi(1)=P$. From the construction of $\xi$ we get:

$$
\mathcal{H}^{1}(\xi([0,1])) \leq \liminf \mathcal{H}^{1}\left(\xi_{n}([0,1])\right)=k .
$$

Since $\mathcal{H}^{1}(K)<\infty$ and since the arcs $\xi_{n}$ converge to $\xi$ in $K$ we shall have

$$
\mathcal{H}^{1}\left(\xi_{n}([0,1]) \backslash \xi([0,1])\right) \rightarrow 0 .
$$

Consequently, for large $n$ there are two points $\mu_{n}$ and $\nu_{n}$ in $\xi_{n}([0,1]) \cap$ $\xi([0,1])$ such that $d_{K}\left(\hat{M}_{n}, \mu_{n}\right)<k / 8$ and $d_{K}\left(\hat{N}_{n}, \nu_{n}\right)<k / 8$. Since, $\mu_{n}$ and $\nu_{n}$ are two points of the closed curve with length at most $k$ defined by $\xi$, we have $d_{K}\left(\mu_{n}, \nu_{n}\right)<k / 2$. Consequently

$$
d_{K}\left(\hat{M}_{n}, \hat{N}_{n}\right)<3 k / 4
$$

which contradicts the relation

$$
d_{K}\left(\hat{M}_{n}, \hat{N}_{n}\right)=\varepsilon_{n} d_{K_{n}}\left(M_{n}, N_{n}\right)=\varepsilon_{n} l_{n} \rightarrow k \quad \text { as } n \rightarrow+\infty .
$$

This contradiction shows that $\varepsilon_{n} l_{n} \rightarrow 0$ and we may apply theorem 2.3 and get the convergence of a blow-up subsequence $\left(v_{n}, G_{n}\right)$ to some $\left(v_{0}, G_{0}\right)$. We consider arcs

$$
\zeta_{n}:[0,1] \rightarrow \mathbf{R}^{2}, \quad \zeta_{n}(t)=\frac{1}{l_{n}} \psi_{n}\left(l_{n} t\right) .
$$

For a subsequence, the arcs $\zeta_{n}$ with length 1 converge uniformly to a an $\operatorname{arc} \zeta$ with length at most 1 and such that $\zeta(0)=\zeta(1)=0$. Thus, $\zeta$ defines a closed curve in $G_{0}$. Since $\left(v_{0}, G_{0}\right)$ is a global-minimizer, the interior of the domain defined by $\zeta$ must be empty (see lemma 4.8). Therefore every point of $\zeta([0,1])$ is reached twice by $\zeta$. Since $\zeta_{n}$ is a Jordan arc with length 1 we get

$$
\mathcal{H}^{1}(\zeta([0,1])) \leq \frac{1}{2}
$$

This can be written as $\mathcal{H}^{1}(\zeta([0,1])) \leq \mathcal{H}^{1}\left(\zeta_{n}([0,1])\right)-1 / 2$. The argument of the proof of theorem 2.2 gives then: for almost all $r>1 / 2$ :

$$
J_{B_{r}}^{0}\left(v_{0}, K_{v_{0}}\right) \leq \liminf J_{B_{r}}^{\varepsilon_{n} l_{n}}\left(v_{n}, \tilde{K}_{n}\right)-\frac{1}{2}
$$

which contradicts the equality (2.8). This completes the proof by contradiction of the lemma. 
Notice that the proof of lemma 2.8 gives immediately that any connected component of $K$ is a chord-arc set:

Lemma 2.9. - There is a constant $C$ such that if $M$ and $N$ are in the same connected component of $K$ then

$$
d_{K}(M, N) \leq C d(M, N)
$$

where $d(M, N)$ denotes the euclidean distance.

\section{A MONOTONICITY FORMULA}

\subsection{Global-minimizers of $J^{0}$}

To characterize the global-minimizers of $J^{0}$, introduced in the previous section, we prove a monotonicity formula

THEOREM 3.1. - Let $(u, K)$ be a global-minimizer and let $\phi(r)=$ $\int_{B_{r} \backslash K}\|\nabla u\|^{2}$. If $K$ is connected then $r \mapsto \frac{\phi(r)}{r}$ is a non-decreasing function of $r$. If $\frac{\phi(r)}{r}$ is constant, then in some polar coordinates $(r, \theta)$ we have:

$$
u(r, \theta)=C \sqrt{r} \cos \frac{\theta}{2} \quad \text { for } \theta \in[0,2 \pi]
$$

and $K$ is the half axis $\{\theta=0\}$.

Remark 3.2. - If $(u, K)$ is defined in a ball $B_{R}, K$ is a closed connected set and $u$ minimizes $\int_{B_{R} \backslash K}\|\nabla u\|^{2}$ under Dirichlet conditions $u=u_{0}$ on $\partial B_{R}$ then the conclusion of theorem 3.1 holds for $r \in(0, R)$.

Monotonicity formulas of another type have been used in the study of two-phase free-boundary problems (see for instance [1]). The proof of theorem 3.1 follows a series of lemmas and propositions. We want to compare the two integrals $\int_{B_{r}}\|\nabla u\|^{2}$ and $r \int_{\partial B_{r} \backslash K}\|\nabla u\|^{2}$ which appears in the derivative of $\frac{\phi(r)}{r}$. We first state a Green formula

LEMMA 3.3. - Let $(u, K)$ be a global-minimizer, then for almost all $r$ :

$$
\int_{B_{r}}\|\nabla u\|^{2}=\int_{\partial B_{r} \backslash K} u \frac{\partial u}{\partial \nu} .
$$

This lemma is analogous to lemma 3.1 in [6]. The reader is referred to [6] for the detailed proof. 
Proposition 3.4. - Let $(u, K)$ be a global-minimizer. If $K$ is connected then for almost every $r$

$$
r \int_{\partial B_{r} \backslash K}\|\nabla u\|^{2} \geq \int_{\partial B_{r} \backslash K} u \frac{\partial u}{\partial \nu} .
$$

Proof. - The proof of this proposition is based on an estimate of $\int_{\partial B_{r} \backslash K}\left|u_{\tau}\right|^{2}$ where $u_{\tau}$ is the tangential derivative on the sphere $\partial B_{r}$. We know that for almost every $r, \partial B_{r} \cap K$ is a finite set and $\int_{\partial B_{r} \backslash S}\|\nabla u\|^{2}<$ $+\infty$ [6]. Therefore, we will prove the inequality under the assumption that $\partial B_{r} \cap K$ is a finite set $\left\{y_{1}, . ., y_{k}\right\}$. In polar coordinates, $y_{i}$ has coordinates $\left(r, \theta_{i}\right)$ and we may assume that $0 \leq \theta_{1}<\theta_{2}<. .<\theta_{k}<2 \pi$. We introduce $\theta_{k+1}=\theta_{1}+2 \pi$ (the point $y_{k+1}$ with coordinates $\left(r, \theta_{k+1}\right)$ coincides with $\left.y_{1}\right)$. We now evaluate the integral of $\|\nabla u\|^{2}$ on each arc $y_{i} y_{i+1}$ :

$$
\begin{aligned}
\int_{y_{i} \bar{y}_{i+1}}\|\nabla u\|^{2} & =r \int_{\theta_{i}}^{\theta_{i+1}}\|\nabla u\|^{2} d \theta \\
& =r \int_{\theta_{i}}^{\theta_{i+1}}\left(\left|u_{r}\right|^{2}+\frac{1}{r^{2}}\left|u_{\theta}\right|^{2}\right) d \theta \\
& \geq 2 r\left(\int_{\theta_{i}}^{\theta_{i+1}}\left|u_{r}\right|^{2}\right)^{1 / 2}\left(\frac{1}{r^{2}} \int_{\theta_{i}}^{\theta_{i+1}}\left|u_{\theta}\right|^{2}\right)^{1 / 2}
\end{aligned}
$$

Here, $u_{r}$ and $u_{\theta}$ denotes the radial and azimuthal partial derivatives. Introducing the average value $\bar{u}_{i}=\frac{1}{\theta_{i+1}-\theta_{i}} \int_{\theta_{i}}^{\theta_{i+1}} u$ of $u$ on the arc $y_{i} \widehat{y_{i+1}}$ we may use the following lemma which is a straightforward consequence of a theorem of Wirtinger (see [15] theorem 258):

LEMMA 3.5. - Let $f \in H^{1}([-\pi, \pi], \mathbf{R})$ and let $\bar{f}=\frac{1}{2 \pi} \int_{-\pi}^{\pi} f$ then

$$
\frac{1}{4} \int_{-\pi}^{\pi}(f-\bar{f})^{2} d x<\int_{-\pi}^{\pi}\left(f^{\prime}\right)^{2} d x
$$

unless $f(x)=a+b \sin \frac{x}{2}$.

By a straightforward change of variables, we get

$$
\int_{\theta_{i}}^{\theta_{i+1}}\left|u_{\theta}\right|^{2} \geq\left(\frac{2 \pi}{\theta_{i+1}-\theta_{i}}\right)^{2} \frac{1}{4} \int_{\theta_{i}}^{\theta_{i+1}}\left(u-\bar{u}_{i}\right)^{2} .
$$

Using this together with (3.1) we deduce:

$$
\begin{aligned}
\int_{y_{i} y_{i+1}}\|\nabla u\|^{2} & \geq 2 r\left(\int_{\theta_{i}}^{\theta_{i+1}}\left|u_{r}\right|^{2}\right)^{1 / 2}\left(\frac{1}{4 r^{2}} \int_{\theta_{i}}^{\theta_{i+1}}\left(u-\bar{u}_{i}\right)^{2}\right)^{1 / 2} \\
& \geq \int_{\theta_{i}}^{\theta_{i+1}} u_{r}\left(u-\bar{u}_{i}\right),
\end{aligned}
$$


that is

$$
\int_{y_{i} \hat{y}_{i+1}}\|\nabla u\|^{2} \geq \frac{1}{r} \int_{y_{i} \widehat{y}_{i+1}} u_{\nu}\left(u-\bar{u}_{i}\right) .
$$

This inequality was derived without any particular assumptions on $u$ and $K$.

Now, if $K$ is connected then the set $y_{i} \widehat{y_{i}+1}$ defines a bounded domain $\mathcal{D}$ of boundary $\partial \mathcal{D}=F \cup y_{i} \widehat{y_{i+1}}$ where $F \subset K$. Then, by a similar argument as in lemma 3.1 [6] we observe that since $\bar{u}_{i}$ is a constant, $\int_{\partial \mathcal{D}} u_{\nu} \bar{u}_{i}=\int_{\mathcal{D}} \nabla u \nabla \bar{u}_{i}=0$. Moreover, $u$ satisfies Neumann boundary conditions on $F$ and then $\int_{\partial \mathcal{D}} u_{\nu} \bar{u}_{i}=\int_{y_{i} y_{i+1}} u_{\nu} \bar{u}_{i}$. Consequently if $K$ is connected (or more generally if $y_{1}, \ldots, y_{k}$ belongs to the same connected component of $K$ ) we have $\int_{y_{i} y_{i+1}} u_{\nu} \bar{u}_{i}=0$ which gives the inequality:

$$
\int_{y_{i} \hat{y}_{i+1}}\|\nabla u\|^{2} \geq \frac{1}{r} \int_{y_{i} \widehat{y}_{i+1}} u_{\nu} u .
$$

Summing over all $i=1, . ., k$ this reads

$$
\int_{\partial B_{r} \backslash K}\|\nabla u\|^{2} \geq \frac{1}{r} \int_{\partial B_{r} \backslash K} u_{\nu} u
$$

which is the conclusion of proposition 3.4.

Proof of theorem 3.1. -

Lemma 3.3 and proposition 3.4 implies

$$
r \phi^{\prime}(r) \geq \phi(r) \text { a.e. }
$$

which is $\left(\frac{\phi(r)}{r}\right)^{\prime} \geq 0$.

The second part of the theorem is concerned with the situations where $\left(\frac{\phi(r)}{r}\right)^{\prime} \equiv 0$. If this occurs then the inequality in (3.5) is in fact an equality for almost every $r$. Consequently, the inequality given in lemma 3.5 is an equality and there are three functions $\alpha(r), \beta(r)$ and $\theta(r)$ such that for a.e. $r$ and for $\theta \in[\theta(r), \theta(r)+2 \pi) u$ is given by $u(r, \theta)=\beta(r)+\alpha(r) \cos \left(\frac{\theta-\theta(r)}{2}\right)$. Since $u$ is harmonic in $\mathbf{R}^{2} \backslash K$ we get $\alpha(r)=\alpha_{0} \sqrt{r}, \beta(r)=\beta_{0}$ and $\theta(r)=\theta_{0}$. This completes the proof of theorem 3.1 . 
We now study global-minimizers defined in a half-space $\mathbf{R}^{+} \times \mathbf{R}$. In this case, $\theta_{i+1}-\theta_{i} \leq \pi$ in (3.2) and it is straightforward to prove the

Corollary 3.6. - If $(u, K)$ is a global-minimizer in $\mathbf{R}^{+} \times \mathbf{R}$ such that $K \cup \partial\left(\mathbf{R}^{+} \times \mathbf{R}\right)$ is connected then $r \mapsto \frac{\phi(r)}{r^{2}}$ is a nondecreasing function of $r$.

Remark 3.7. - If $U=\{y>f(x)\}$ is a Lipschitz domain $(f(0)=0)$ and if $(u, K)$ is a global-minimizer in $U$ such that $K \cup \partial U$ is connected then there is a $\alpha \in(1,2]$ such that $r \mapsto \frac{\phi(r)}{r^{\alpha}}$ is a nondecreasing function of $r$.

\subsection{Minimizers of $J$}

We consider now a minimizer $(u, K)$ of the Mumford-Shah functional

$$
J(u, K)=\int_{\Omega \backslash K}\|\nabla u\|^{2}+\int_{\Omega}(u-g)^{2}+\mathcal{H}^{1}(K) .
$$

LEMMA 3.8. - Assume that $\omega \subset \Omega$ intersects only one connected component of $K$. Let $U$ be an open set $U \subset \subset \omega$. There is a constant $C>0$ and a real $r_{0}$ such that for all $P \in U$ we have:

$$
\text { for a.e. } r \in\left(0, r_{0}\right), \int_{\partial B_{r}(P) \backslash K}\|\nabla u\|^{2} \geq \frac{1}{r} \int_{B_{r}(P) \backslash K}\|\nabla u\|^{2}-C r
$$

Proof. - The lemma will be derived from inequality (3.3) by an estimation of $\int_{y_{i} y_{i+1}} u_{\nu} \bar{u}_{i}$. Since $y_{i}$ and $y_{i+1}$ are in the same connected component there is an arc of minimum length $\psi_{i}$ connecting $y_{i}$ to $y_{i+1}$. Lemma 2.9 implies that $\mathcal{H}^{1}\left(\psi_{i}([0,1])\right) \leq C d\left(y_{i}, y_{i+1}\right) \leq C^{\prime} r$. Therefore, integrating in the domain $\omega_{i}$ defined by $\psi_{i}([0,1])$ and the arc $y_{i} y_{i+1}$ we get

$$
\begin{aligned}
\int_{y_{i} \bar{y}_{i+1}} u_{\nu} \bar{u}_{i} & =\int_{\omega_{i} \backslash K} \nabla u \nabla \bar{u}_{i}+\int_{\omega_{i} \backslash K} \Delta u \bar{u}_{i} \\
& =\int_{\omega_{i}}(u-g) \bar{u}_{i}
\end{aligned}
$$

Since $\mathcal{H}^{1}\left(\partial \omega_{i}\right)<\left(C^{\prime}+2 \pi\right) r$ we get, by isoperimetric inequality, $\left|\omega_{i}\right|<C^{\prime \prime} r^{2}$ and then $\left|\int_{\omega_{i}}(u-g) \bar{u}_{i}\right| \leq C^{\prime \prime} r^{2}$. Inequality (3.3) gives then the conclusion of the lemma. 


\section{CHARACTERIZATION OF GLOBAL-MINIMIZERS}

The main theorem of this section is:

THEOREM 4.1. - If $(u, K)$ is a global-minimizer such that $K$ is connected then $(u, K)$ is one of the following:

(i) $K$ is empty and $u$ is constant.

(ii) $K$ is a straight line defining two half-planes and $u$ is constant on each half-plane.

(iii) $K$ is the union of three half lines with angle $2 \pi / 3$ and $u$ is constant on each sector.

(iv) in a polar set of coordinates $u(r, \theta)=C \pm \sqrt{\frac{2}{\pi}} \sqrt{r} \cos \frac{\theta}{2}$ for $\theta \in[0,2 \pi)$ and $K$ is the half axis $\theta=0$.

Proof. - The proof relies on a careful use of the monotonicity formula given in the previous section.

LEMMA 4.2. - If $(u, K)$ is a global-minimizer such that $\frac{\phi(r)}{r}$ is constant then either

(i) $\frac{\phi(r)}{r} \equiv 1$ and in a polar set of coordinates $u(r, \theta)=C \pm \sqrt{\frac{2}{\pi}} \sqrt{r} \cos \frac{\theta}{2}$ for $\theta \in[0,2 \pi)$.

(ii) or $\frac{\phi(r)}{r} \equiv 0$.

Proof. - The determination of the constant $\sqrt{\frac{2}{\pi}}$ can be found in [19]. The lemma is then a straightforward consequence of theorem 3.1.

To prove theorem 4.1 we will show that $\frac{\phi(r)}{r}$ is a constant for a properly chosen origin. For this purpose we study the limits $\lim _{+\infty} \frac{\phi(r)}{r}$ and $\lim _{0} \frac{\phi(r)}{r}$ using a blow-up and blow-down technique. The blow-up is defined as in section 2 , that is,

$$
\begin{aligned}
U_{\varepsilon}(X) & =\frac{u(\varepsilon X)}{\sqrt{\varepsilon}} \quad \text { and } \quad u_{\varepsilon}(X)=\frac{u(\varepsilon X)-c_{\varepsilon}(X)}{\sqrt{\varepsilon}}, \\
K_{\varepsilon} & =\left\{X \in \mathbf{R}^{2}, \varepsilon X \in K\right\}
\end{aligned}
$$

and the blow-down is given by

$$
\begin{aligned}
& u_{l}(X)=\frac{u(l X)}{\sqrt{l}}, \\
& K_{l}=\left\{X \in \mathbf{R}^{2}, l X \in K\right\}
\end{aligned}
$$

where $\varepsilon \rightarrow 0$ and $l \rightarrow+\infty$. 
From theorem 2.2 we deduce the,

COROLlaRY 4.3. - Let $(u, K)$ be a global-minimizer then

$\left({ }^{*}\right)$ a blow-up subsequence $u_{\varepsilon_{q}}, K_{\varepsilon_{q}}$ (i.e. $\varepsilon_{q} \rightarrow 0$ as $q \in \mathbf{N}$ goes to $\infty$ ) converges to a global-minimizer $\left(u_{0}, K_{0}\right)$,

(**) a blow-down subsequence $u_{l_{p}}, K_{l_{p}}$ (i.e. $l_{p} \rightarrow \infty$ as $p \in \mathbf{N}$ goes to $\infty)$ converges to a global-minimizer $\left(u_{\infty}, K_{\infty}\right)$.

The convergences are to be understood in the same sense as stated in theorem 2.2. We can deduce then

$$
\begin{aligned}
& \text { LEMMA 4.4. }- \text { Let } \phi_{0}(r)=\int_{B_{r}}\left\|\nabla u_{0}\right\|^{2} \text { and } \phi_{\infty}(r)=\int_{B_{r}}\left\|\nabla u_{\infty}\right\|^{2} \text {. Then } \\
& \qquad \frac{\phi_{0}(r)}{r} \equiv \lim _{0} \frac{\phi(\rho)}{\rho} \quad \text { and } \quad \frac{\phi_{\infty}(r)}{r} \equiv \lim _{\infty} \frac{\phi(\rho)}{\rho}
\end{aligned}
$$

With lemma 4.2 this implies,

LeMma 4.5. - If $(u, K)$ is a global-minimizer such that $K$ is connected then $\lim _{0} \frac{\phi(r)}{r}$ and $\lim _{\infty} \frac{\phi(r)}{r}$ can only take the value 0 or 1 .

Consequently, if $\lim _{\infty} \frac{\phi(r)}{r}=0$ then the monotonicity formula gives $\phi(r) \equiv 0$. Thus, $u$ is a constant in any connected component of $\mathbf{R}^{2} \backslash K_{0}$ and we are reduced to minimizing $\mathcal{H}^{1}\left(K_{0}\right)$ under the condition (ii) of definition 2.1 which gives situations (i), (ii) or (iii) of theorem 4.1.

Let $(u, K)$ be a minimizer of the Mumford-Shah functional $J(u, K)$ or $J^{0}(u, K)$. Assume that $K$ has a finite number of connected components. We consider the blow-up at a point $P: u_{\varepsilon}(X)=\frac{u(P+\varepsilon X)-c_{\varepsilon}(X)}{\sqrt{\varepsilon}}$. The next lemma gives additional information about the way blow-ups converge in case (i), (ii), (iii).

LEMMA 4.6. - If a blow-up limit at a point $P$ (i.e. the limit of a subsequence $\left(u_{\varepsilon_{n}}, K_{\varepsilon_{n}}\right)$ is of type (i), (ii) or (iii) then for any other subsequence the blow-up limit at $P$ is of the same type.

Proof. - We study the function

$$
\xi: r \mapsto \frac{\int_{B_{r}(P) \backslash K}\|\nabla u\|^{2}}{r}
$$

Under the hypotheses of lemma 4.6, lemma 3.8 implies that $\xi^{\prime}(r) \geq-C$ for $r$ small. Then, $\xi$ has a limit $l$ as $r \rightarrow 0$.

We claim that for any converging subsequence, the blow-up limit $\left(u_{0}, K_{0}\right)$ is such that the ratio $\frac{\int_{B_{r}(0) \backslash K}\left\|\nabla u_{0}\right\|^{2}}{r}$ is constant and equal to $l$. This is a straightforward consequence of equations (2.12), (2.14) and (2.8). Under the 
assumptions of lemma 4.6, this implies that $l=0$ and that every blow-up limit is such that $\frac{\int_{B_{r}(0) \backslash K}\left\|\nabla u_{0}\right\|^{2}}{r}$ is zero. Therefore, every blow-up limit cannot be of type (iv), it is of type (i), (ii) or (iii). This is a very strong constraint on the solutions.

Assume first that a blow-up limit is of type (i). We recall [6] that there is a constant $C$ such that if $\mathcal{H}^{1}\left(K \cap B_{r}\right)<C r$ then $K \cap B_{\frac{r}{2}}=\emptyset$. If the blowup limit is of type (i), then (2.14) implies that $\liminf \mathcal{H}^{1}\left(K_{\varepsilon} \cap B_{1}\right)=0$. Therefore there is $\varepsilon$ such that $\mathcal{H}^{1}\left(K_{\varepsilon} \cap B_{1}\right)<C$, that is, $\mathcal{H}^{1}\left(K \cap B_{\varepsilon}\right)<C \varepsilon$. This implies that $K \cap B_{\frac{\varepsilon}{2}}=\emptyset$ and then every blow-up limit is of type (i).

It remains to understand what happens when the blow-up limit is of type (ii) or (iii). We argue by contradiction and assume that there are two blow-up sequences $\varepsilon_{p}$ and $\eta_{p}$ such that $\left(u_{\varepsilon_{p}}, K_{\varepsilon_{p}}\right)$ converges to a type (ii) and $\left(u_{\eta_{p}}, K_{\eta_{p}}\right)$ converges to a type (iii). We may assume that $\varepsilon_{p} \geq \eta_{p}>\varepsilon_{p+1}$. Notice that this implies the convergence $\frac{\eta_{p}}{\varepsilon_{p}} \rightarrow 0$ (if not, the blow-up limit should be at the same time a type (ii) and a type (iii)). We will now prove that we can construct a blow-up sequence inbetween $\varepsilon_{p}$ and $\eta_{p}$ which converges to a global-minimizer which cannot be of one of the types of theorem 4.1.

Let us denote by $k^{2}$ the limit edge set of type (ii). It is a line passing through the origin 0 . Let $M$ be the triple point of the limit edge set of type (iii). We denote this set by $M+k^{3}$ where $k^{3}$ is the union of three half lines meeting at the origin and making $2 \pi / 3$ angles. For any $R$ we have the convergence: $K_{\eta_{p}} \cap B_{R} \rightarrow\left(M+k^{3}\right) \cap B_{R}$ in the Hausdorff metric. We may choose a sequence $l_{p}$ going slowly to $+\infty$ such that $l_{p} \eta_{p}<\varepsilon_{p}$ and for all $R, K_{l_{p} \eta_{p}} \cap B_{R} \rightarrow k^{3} \cap B_{R}$. For simplicity, we will assume in the following that the sequence $\eta_{p}$ is chosen such that $K_{\eta_{p}} \cap B_{R} \rightarrow k^{3} \cap B_{R}$. Theorem 2.2 implies that $\mathcal{H}^{1}\left(B_{R} \cap K_{\eta_{p}}\right) \rightarrow 3 R$ uniformly in any compact interval. For the same reasons $\mathcal{H}^{1}\left(B_{R} \cap K_{\varepsilon_{p}}\right) \rightarrow 2 R$ uniformly in any compact interval. We introduce $R_{p}=\inf \left\{R \geq 1, \mathcal{H}^{1}\left(B_{R} \cap K_{\eta_{p}}\right) \leq 2.5 R\right\}$. Since $\varepsilon_{p}>\eta_{p}$, we have $R_{p}<\frac{\varepsilon_{p}}{\eta_{p}}$ and $R_{p} \rightarrow+\infty$. We are interested in the blow-up sequence $\left(u_{\zeta_{p}}, K_{\zeta_{p}}\right)$ for $\zeta_{p}=R_{p} \varepsilon_{p}$. Since $\mathcal{H}^{1}\left(B_{R} \cap K\right)$ is an increasing function of $R$, the definition of $R_{p}$ and $\zeta_{p}$ gives

$$
\forall R \in\left(1 / R_{p}, 1\right), 2.5 R \leq \mathcal{H}^{1}\left(B_{R} \cap K_{\zeta_{p}}\right) \leq 2 . \dot{5} .
$$

A subsequence of $\left(u_{\zeta_{p}}, K_{\zeta_{p}}\right)$ converges to some $\left(u_{0}, K_{0}\right)$ such that

$$
\forall R \in(0,1), \quad 2.5 R \leq \mathcal{H}^{1}\left(B_{R} \cap K_{0}\right) \leq 2.5
$$

Vol. $13, \mathrm{n}^{\circ} 4-1996$. 
None of the global-minimizers of theorem 4.1 satisfies this relation. This contradiction completes the proof of lemma 4.6.

So far, we have proved that either we are in case (i), (ii), (iii) of theorem 4.1 , or we have $\lim _{\infty} \frac{\phi(r)}{r}=1$.

PROPOSITION 4.7. - If $\lim _{\infty} \frac{\phi(r)}{r}=1$ then there is a point $P$ such that if we denote $\phi_{P}(r)=\int_{B_{R}(P)}\|\nabla u\|^{2}$ then

$$
\lim _{0} \frac{\phi_{P}(r)}{r}=1
$$

We will say that $P$ is a crack-tip for the edge set $K$.

Proof. - We argue by contradiction. If the conclusion of the proposition is not true then for every point $P$ the blow-up limit at $P$ gives situations (i), (ii) or (iii) of theorem 4.1. We consider a point $P \in K$ and Jordan arcs in $K$ parameterized by arc length $\psi:(a, b) \rightarrow K$ such that $\psi(0)=P$, ( $\psi$ is one to one). We consider maximal Jordan arcs passing through $P$ : $\psi$ is such that if $\tilde{\psi}:(\tilde{a}, \tilde{b}) \rightarrow K$ is a Jordan arc in $K$ parameterized by arc length and $(a, b) \subset(\tilde{a}, \tilde{b})$ and $\tilde{\psi}_{/(a, b)}=\psi$ then $(a, b)=(\tilde{a}, \tilde{b})$ and $\tilde{\psi}=\psi$. Since $K$ is a 1 -dimensional Hausdorff connected set, it is arcwise connected. By assumption, $K$ is unbounded and then, there is a maximal Jordan arc $\psi:(a, b) \rightarrow K$ such that $\psi(0)=P$ and such that $a-b$ is arbitrary large. If $(a, b)=\mathbf{R}$ then $\forall r>0, \mathcal{H}^{1}\left(K \cap B_{r}(P) \geq 2 r\right.$ which contradicts the fact that the blow-down is of type (iv). Therefore we may assume that $b<+\infty$. and we may introduce $N=\psi(b)$.

* If $N \in \psi([a, b))(N=\psi(c))$ then $\psi:[c, b] \rightarrow K$ defines a closed Jordan curve defining a bounded connected component of $\mathbf{R}^{2} \backslash K$. This contradicts lemma 4.8 below.

* If $\psi(b) \notin \psi([a, b))$ then the blow-up limit at $N$ is either a type (ii) or a type (iii).

Assume that one blow-up limit is of type (ii). Lemma 4.6 says that every blow-up limit is of type (ii). This reads: $\forall \varepsilon>0 \exists r_{0}$, $\forall r<r_{0}, \exists L^{r}, L^{r}$ is a straight line passing through $N$ such that $K \cap B_{r}(N) \subset\left\{X \in \mathbf{R}^{2}, d\left(X, L^{r}\right)<\varepsilon r\right\}$. Let $\varepsilon=1 / 2$. Then, $\partial B_{r}(N) \cap\left\{X \in \mathbf{R}^{2}, d\left(X, L^{r}\right)<r / 2\right\}$ is made of two components $C_{1}^{r}$ and $C_{2}^{r}$. There exists $r_{1}$ such that for $r<r_{1}, \psi((a, b)) \cap C_{1}^{r} \neq \emptyset$ implies $\psi((a, b)) \cap C_{2}^{r}=\emptyset$. Indeed, otherwise this would imply (for $r$ arbitrary small) that $\mathcal{H}^{1}\left(K \cap B_{r}(N)\right) \geq(1+\sqrt{3}) r$. However, since the 
blow-up limit is of type (ii), we should have $\lim _{r \rightarrow 0} \frac{\mathcal{H}^{1}\left(K \cap B_{r}(N)\right)}{C_{r}}=2$. Therefore we will assume that $\psi((a, b))$ intersects only $\underset{C_{1}^{r}}{r}$ for $r<r_{1}$. Since the blow-up limit is a line, there is a sequence $N_{n} \in K \cap C_{2}^{r_{n}}$, $\left(r_{n}=\left\|N_{n}-N\right\|>0\right)$ converging to $N$. Let $\xi_{n}:(b, c) \rightarrow K$ be an arc of minimal length connecting $N$ to $N_{n}$ in $K$. This is a Jordan arc and we may assume that it is parameterized by arc length. Lemma 2.9 implies that its length goes to 0 . Then for $n$ large enough $\xi_{n}(b, c) \subset B_{r_{1}}(N)$ and we can prove easily by connexity that $\xi_{n}(t) \in C_{r_{n}(t)}^{2}\left(\right.$ where $\left.r_{n}(t)=\left\|\xi_{n}(t)-N\right\|\right)$ for all $t \in(b, c)$. Consequently $\xi_{n}(b, c) \cap \psi(a, b)=\emptyset$. We may then extend $\psi$ on $(a, c)$ by $\xi_{n}$ and contradict the maximality of $\psi$.

If the blow-up limit at $N$ is of type (iii) (union of 3 half lines $D_{i}$ ) the same argument can be carried out with two connected components of $\partial B_{r} \cap\left\{X \in \mathbf{R}^{2}, \exists i, d\left(X, D_{i}\right)<\varepsilon r\right\}$ instead of $C_{1}^{r}$ and $C_{2}^{r}$ and give again a contradiction. This completes the proof of proposition 4.7.

End of the proof of theorem 4.1. - We have proved that $\lim _{\infty} \frac{\phi(r)}{r}$ can only take the value 0 or 1 (lemma 4.5). If $\lim _{\infty} \frac{\phi(r)}{r}=0$ then we get (i), (ii) or (iii) in theorem 4.1. If $\lim _{\infty} \frac{\phi(r)}{r}=1$ then there is a $P$ such that $\frac{\phi_{P}(r)}{r}$ is constant and equal to 1 . This gives the situation (iv) of theorem 4.1 whose proof is completed.

We have been using in the previous proof the fact that $K \backslash \mathbf{R}^{2}$ cannot have a bounded connected component.

LEMMA 4.8. - If $(u, K)$ is a global-minimizer, then $\mathbf{R}^{2} \backslash K$ cannot have a bounded connected component.

Proof. - Notice that by an obvious excision argument, any bounded connected component of $\mathbf{R}^{2} \backslash K$ is convex. By contradiction, assume that $\mathbf{R}^{2} \backslash K$ has a bounded convex connected component $\mathcal{O}$. Now, $u$ is necessary a constant in the bounded connected component $\mathcal{O}$. Let $A$ be a regular point of $\partial \mathcal{O}$. The blow-up at $A$ is a line (situation (ii)). There is a limit value of $u$ on both side of $K$ at $A$. We may choose a compact perturbation of $u$ inside $\mathcal{O}$ such that those two values are identical. Then the blow-up at $A$ can be proceeded with a function $c$ constant in the whole plane. Therefore, the blow-up limit has to be a global-minimizer with respect to any compact perturbation (the assumption (ii) of definition 2.1 is not required). Here, the blow-up limit $\left(u_{0}, K_{0}\right)$ is such that $K_{0}$ is a line and $u_{0} \equiv 0$ in $\mathbf{R}^{2} \backslash K_{0}$. Consequently for any ball $B_{R}$ one can take $\tilde{K}_{0}=K_{0} \backslash B_{R}$ and $\tilde{u} \equiv 0$ in $\mathbf{R}^{2} \backslash \tilde{K}_{0}$ and reduce the $J^{0}$ energy. This contradicts the minimality of $\left(u_{0}, K_{0}\right)$. 


\section{FINITE NUMBER OF PIECES}

In this section we will prove that any isolated connected component of $K$ is made of a finite number of Jordan arcs.

THEOREM 5.1. - Let $\Omega$ be a Lipschitz domain and $G$ be an isolated connected component of $K$. Then,

$$
G=\bigcup_{i=1}^{k} \psi_{i}([0,1])
$$

where $\psi_{i}, i=1, . ., k$, are one to one Lipschitz continuous functions and $\psi_{i}((0,1)) \cap \psi_{j}((0,1))=\emptyset$ if $i \neq j$.

Let $P$ be a point in a neighborhood of $G$. Theorem 2.2 and lemma 2.8 ensure that a subsequence of the blow-up sequence of $(u, K)$ at $P$ converges to a global-minimizer which satisfies the hypotheses of theorem 4.1. As noted in remark 2.4 there may be several blow-up limits depending of the chosen subsequence. However we have

Proposition 5.2. - All blow-up limits at $P$ are of the same type (that is, (i), (ii), (iii) and (iv) of theorem 4.1). Type (i) is obtained when $P \notin K$, for type (ii) $P$ will be called a flat point, for type (iii) $P$ is a triple point and for type (iv) $P$ is a crack-tip.

Proof. - We first notice that lemma 2.8 implies that any blow-up limit is such that the edge set is connected. Consequently, theorem 4.1 implies that the blow-up limit has to be of type (i), (ii), (iii) or (iv). Lemma 4.6 gives then the conclusion.

Proof of theorem 5.1. - Let us first assume that $G \subset \subset \Omega$. We know (proposition 5.2) that any point of $K$ is a flat point or a triple point or a crack-tip. Let us prove that there is a finite number of triple points. By contradiction, assume that there is a sequence $T_{n}$ of distinct triple points in $G$. By compactness we may assume that $T_{n}$ converges to a point $P$ of $G$. We introduce the blow-up sequence $u_{\varepsilon_{n}}(X)=\frac{u\left(T_{n}+\varepsilon_{n} r(X)\right)-c_{\varepsilon_{n}}(X)}{\sqrt{\varepsilon_{n}}}$, $K_{\varepsilon_{n}}=\left\{X \in \mathbf{R}^{2}, T_{n}+\varepsilon_{n} r(X) \in K\right\}$ where $\varepsilon_{n}=\left\|T_{n+1}-T_{n}\right\|$ and $r$ is the rotation which sends $e_{x}$ on $\frac{T_{n+1}-T_{n}}{\varepsilon_{n}}$. For this transformation, $K_{\varepsilon_{n}}$ has a triple point at the origin and at the point $A=(1,0)$. A subsequence of $\left(u_{\varepsilon_{n}}, K_{\varepsilon_{n}}\right)$ converges to a global-minimizer $\left(u_{0}, K_{0}\right)$ of $J^{0}$. The origin and $A$ are in the set $K_{0}$ and at least one of the two is not a triple point (theorem 4.1). Assume that the origin is not a triple point. It is then a crack-tip or a flat point. Therefore,

$$
\left.\mathcal{H}^{1}\left(K_{\varepsilon_{n}} \cap B_{r}(0)\right) \underset{n \rightarrow+\infty}{\rightarrow} r \text { (resp. } 2 r\right) \text { uniformly in } r \in(0,1) \text {. }
$$


Since $T_{n}$ is a triple point we have

$$
\lim _{r \rightarrow 0} \frac{\mathcal{H}^{1}\left(K_{\varepsilon_{n}} \cap B_{r}\right)}{r}=3 .
$$

As in the proof of lemma 4.6 we introduce $R_{n}=\inf (r>0$, $\left.\mathcal{H}^{1}\left(K_{\varepsilon_{n}} \cap B_{r}\right) \leq 2.5 r\right\}$. We notice that $R_{n} \rightarrow 0$ and for $\eta_{n}=R_{n} \varepsilon_{n}$ we consider the blow-up $u_{\eta_{n}}(X)=\frac{u\left(T_{n}+\eta_{n} r(X)\right)-c_{\eta_{n}}(X)}{\sqrt{\eta_{n}}}, K_{\eta_{n}}=\left\{X \in \mathbf{R}^{2}\right.$, $\left.T_{n}+\eta_{n} r(X) \in K\right\}$. Then,

$$
\forall R \in(0,1), \quad 2.5 R \leq \mathcal{H}^{1}\left(K_{\varepsilon_{n}} \cap B_{r}\right) \leq 2.5
$$

As in lemma 4.6 the blow-up limit satisfies the above inequality and this contradicts the result of theorem 4.1. Consequently, when $G \subset \subset \Omega$, there is a finite number of triple points.

Let us now consider the case where $G$ meets $\partial \Omega$. Assume first that $\Omega$ is regular. We study then a blow-up at a boundary point. This gives a global-minimizer defined in a half-space $\mathbf{R}^{+} \times \mathbf{R}$. From corollary 3.6 and the fact that $\phi(r) \leq C r$ it is obvious that the blow-up limit satisfies: $\phi \equiv 0$ and $K$ is the half line $\mathbf{R}^{+} \times\{0\}$. A similar argument as before shows that there cannot be a sequence of triple points converging to the boundary. If $\Omega$ is only Lipschitz then remark 3.7 gives a similar characterization of blow-up limits ( $\phi \equiv 0, K$ is a half-line ending at the origin, etc.) The same argument as before gives the finite number of triple points.

Similarly, it is even easier to prove that there is at most a finite number of crack-tips.

To conclude the proof of theorem 5.1 we have to prove that we have a finite number of curves. We consider a flat point $P$ of $G$. As in the proof of lemma 4.7, we can prove that there is a Jordan arc parameterized by $\psi:[a, b] \rightarrow K$ of maximal length, passing trough $P$ and such that every point of $\psi((a, b))$ is flat. Then, the endpoints $\psi(a)$ and $\psi(b)$ are either triple points or crack-tips. We note also that for any Jordan arc $\psi^{\prime}:\left[a^{\prime}, b^{\prime}\right] \rightarrow K$, if every point of $\psi\left(\left(a^{\prime}, b^{\prime}\right)\right)$ is flat then either

$$
\psi\left(\left(a^{\prime}, b^{\prime}\right)\right) \cap \psi\left((a, b)=\emptyset \quad \text { or } \quad \psi\left(\left(a^{\prime}, b^{\prime}\right)\right) \subset \psi((a . b)) .\right.
$$

Indeed, consider $\left(t_{1}, t_{2}\right) \subset\left[a^{\prime}, b^{\prime}\right]$ such that $\psi^{\prime}\left(\left(t_{1}, t_{2}\right)\right) \cap \psi((a, b))=\emptyset$ and $\psi^{\prime}\left(t_{1}\right)$ (or $\left.\psi^{\prime}\left(t_{2}\right)\right)$ is in $\psi([a, b])$. Either $\psi^{\prime}\left(t_{1}\right)$ is an endpoint of $\psi([a, b])$ (i.e. coincide with $\psi(a)$ or $\psi(b)$ ) or $\psi^{\prime}\left(t_{1}\right)$ is a triple point. If $\psi^{\prime}\left(t_{1}\right)$ is a triple point then, by construction of $\psi([a, b])$, it is an endpoint: $\psi(a)$ or $\psi(b)$.

Consequently, $G$ is a union of disjoint Jordan arcs connecting a finite family of triple points and crack-tips with each other. The family of 
arcs is then obviously finite $\left(\mathcal{H}^{1}(K)<\infty\right)$. This completes the proof of theorem 5.1 .

Proposition 5.3. - Let $P$ be a point of $K$. Assume that $B_{r}(P)$ intersects only one connected component of $K$. Then

$$
K \cap B_{\frac{r}{2}}(P)=\left(\bigcup_{i=1}^{k} \psi_{i}([0,1])\right) \cap B_{\frac{r}{2}}(P)
$$

where $\psi_{i}$ are one to one Lipschitz continuous functions and $\psi_{i}((0,1)) \cap$ $\psi_{j}((0,1))=\emptyset$ if $i \neq j$.

Proof. - As in the proof of theorem 5.1, there is a finite number of triple points and crack-tips in $B_{\frac{3 r}{4}}(P)$. For each $M \in K \cap B_{\frac{r}{2}}(P)$ there is a Jordan arc $\psi:[a, b] \rightarrow K$ of maximal length, passing trough $M$, such that every point of $\psi((a, b))$ is flat and such that $\psi((a, b)) \subset B_{\frac{3 r}{4}}(P)$. As before those arcs are pairwise disjoint. The endpoints $\psi(a)$ and $\psi(b)$ of this arc are either triple points or crack-tips or points of the boundary $\partial B_{\frac{3 r}{4}}(P)$. If $\psi(a)$ or $\psi(b)$ is in $\partial B_{\frac{3 r}{4}}(P)$ then $\mathcal{H}^{1}(\psi((a, b))) \geq r / 4$. Since $\mathcal{H}^{1}\left(\stackrel{4}{K} \cap B_{r}\right) \leq C r$ there is a finite number of those Jordan arcs intersecting $B_{\frac{r}{2}}(P)$ and ending on $\partial B_{\frac{3 r}{4}}(P)$. We already know (as in theorem 5.1) that there is a finite number of those arcs ending at a triple-point or at a crack-tip. Consequently the family of maximal Jordan arcs made of flat points of $K$ and intersecting $B_{\frac{r}{2}}(P)$ is finite and satisfies the conclusion of the proposition.

\section{REGULARITY $C^{1, \alpha}$}

Let $U$ be a domain, we recall the definition of $C^{1, \alpha}(\bar{U})$.

$$
\begin{gathered}
C^{0, \alpha}(\bar{U})=\left\{f \in C(\bar{U}), \sup _{\substack{x, y \in \bar{U} \\
x \neq y}} \frac{|f(x)-f(y)|}{|x-y|^{\alpha}}<+\infty\right\} \\
C^{m, \alpha}(\bar{U})=\left\{f \in C^{m}(\bar{U}), \quad D^{\beta} f \in C^{0, \alpha}(\bar{U}) \quad \forall \beta \text { with }|\beta|=m\right\}
\end{gathered}
$$

A curve $c$ is $C^{1, \alpha}$ if locally one can find local coordinates $(x, y)$ such that $c \cap B(0, r)$ is a graph $\{y=f(x)\}$ with $f \in C^{1, \alpha}$.

THeOREM 6.1. - Let $(u, K)$ be a minimizer of the Mumford-Shah functional. Let $P$ be a flat point of $K$. Assume that there is an open neighborhood $\omega$ of $P$ such that $\omega$ intersects only one connected component 
of $K$. Then, there is a neighborhood of $P$ in which $K$ is a $C^{1, \alpha}$ arc for all $\alpha<1 / 2$.

Let $\phi_{P}: r \mapsto \int_{B_{r}(P) \backslash K}\|\nabla u\|^{2}$. We will prove a weak monotonicity formula

Lemma 6.2. - Assume that for any $R<R_{0}$ there is a straight line $L_{R}$ passing through $P$ such that $K \cap B_{R}(P) \subset\left\{X \in B_{R}(P), d\left(X, L_{R}\right)<l R\right\}$ where $0<l<1$ is a constant. Assume that only one component of $K$ intersects $B_{R_{0}}$ and that for all $R<R_{0}, K$ intersects both connected components of $\partial B_{R}(P) \cap\left\{X \in B_{R}(P), d\left(X, L_{R}\right)<l R\right\}$, then there is $\beta=\beta(l)$ and a constant $C\left(R_{0}, \beta\right)$ such that

$$
\phi_{P}(R) \leq C R^{\beta}, \quad \forall R<R_{0}
$$

The constant $\beta$ is such that $\beta(l) \rightarrow 2$ as $l \rightarrow 0$.

Proof. - Let $\eta=\sin ^{-1} l$. Inequality (3.2) with $\left|\theta_{i+1}-\theta_{i}\right| \leq \pi+2 \eta$ allows us to adapt the proof of lemma 3.8 and get for $r<R_{0}$,

$$
\int_{\partial B_{r}(P) \backslash K}\|\nabla u\|^{2} \geq \frac{1}{r} \frac{2 \pi}{\pi+2 \eta} \int_{B_{r}(P) \backslash K}\|\nabla u\|^{2}-C r .
$$

We define $\beta=\frac{2 \pi}{\pi+2 \eta}, 1<\beta<2$. Inequality (6.1) reads then

$$
\frac{d}{d r}\left(\frac{\phi_{P}(r)}{r^{\beta}}\right) \geq-C r^{1-\beta}
$$

A direct integration between $R<R_{0}$ and $R_{0}$ gives

$$
\phi_{P}(R) \leq R^{\beta}\left(\frac{\phi_{P}\left(R_{0}\right)}{R_{0}^{\beta}}+\frac{C}{2-\beta} R_{0}^{2-\beta}\right)
$$

which is exactly the conclusion of the lemma with $\beta(l)=\frac{2 \pi}{\pi+2 \sin ^{-1} l}$. Obviously, $1<\beta(l)<2$ and $\beta(l) \rightarrow 2$ as $l \rightarrow 0$.

LEMMA 6.3. - Under the assumptions of lemma 6.2 and for $l<\frac{\sqrt{3}}{2}$ there is a constant $C\left(R_{0}, \beta\right)$ such that for $R<R_{0}$,

$$
\mathcal{H}^{1}\left(K \cap B_{R}(P)\right) \leq 2 R+C R^{\beta}
$$

Proof. - We remark that under the hypotheses of lemma 6.2 and for $l<\frac{\sqrt{3}}{2}$ any blow-up sequence at $P$ will yields a type (ii) blow-up limit Vol. 13, n ${ }^{\circ} 4-1996$. 
(theorem 4.1). This is indeed the only type compatible with the hypotheses. Therefore we know that

$$
\lim _{R \rightarrow 0} \frac{\mathcal{H}^{1}\left(K \cap B_{R}(P)\right)}{R}=2 .
$$

Let $n_{0} \in \mathbf{N}$ be chosen such that $R_{0} \in\left[2^{-n_{0}-1}, 2^{-n_{0}}\right)$. The limit (6.4) and inequality (6.1) imply then the existence of a constant $C_{1}>0$ such that for all $n \geq n_{0}$,

$$
\exists r \in\left[2^{-n}, 2^{-n+1}\right)
$$

such that

$$
\int_{\partial B_{r}(P)}\|\nabla u\|^{2} \leq C_{1} r^{\beta-1} \quad \text { and } \quad \#\left(K \cap B_{r}(P)\right)=2 .
$$

Let $K \cap \partial B_{r}(P)=\left\{y_{1}, y_{2}\right\}$. We introduce a compact perturbation $(\tilde{u}, \tilde{K})$ of $(u, K)$. In $B_{r}(P)$ we replace $K$ by the union of the two segments: $\tilde{K} \cap B_{r}(P)=\left[P, y_{1}\right] \cup\left[P, y_{2}\right]$. Outside $B_{r}(P)$ we define $\tilde{K} \backslash B_{r}(P)=K \backslash B_{r}(P)$. The function $\tilde{u}$ is introduced as: $\tilde{u}=u$ in $\Omega \backslash B_{r}(P)$ and $\tilde{u}$ minimizes the energy $\int_{B_{r}(P) \backslash \tilde{K}}\|\nabla v\|^{2}$ under the Dirichlet condition $v=u$ on $\partial B_{r}(P)$.

We claim that $\int_{B_{r}(P) \backslash \tilde{K}}\|\nabla \tilde{u}\|^{2}$ is less than $C_{2} r^{\beta}$. Indeed $B_{r}(P) \backslash \tilde{K}$ has two sectors $\sigma_{i}$ of angles $\gamma_{i} \pi\left(i=1,2\right.$ and $\left.\gamma_{1}+\gamma_{2}=2\right)$ which can be mapped by a conformal mapping (we identify $\mathbf{R}^{2}$ and $\mathbf{C}$ ) $z \in \mathbf{C} \mapsto \lambda\left(z-z_{P}\right)^{\frac{1}{\gamma_{i}}}$ (where $\lambda \in \mathbf{C},|\lambda|=1$ ) into the half ball $B_{r} \frac{1}{\gamma_{i}} \cap\left(\mathbf{R}+i \mathbf{R}^{+}\right)$. If $v$ is the image of $\tilde{u}$ by this conformal mapping then we have $\frac{\partial v}{\partial \nu}=0$ on the diameter of the half ball. By a reflection we extend $v$ to an harmonic function $w$ on the ball $B_{r} \frac{1}{\gamma_{i}}$. We have then the identity [6]:

$$
\int_{\partial B_{r} \frac{1}{\gamma_{i}}}\left|w_{\nu}\right|^{2}=\int_{\partial B_{r} \frac{1}{\gamma_{i}}}\left|w_{\tau}\right|^{2}
$$

The monotonicity formula (theorem 3.1) gives:

$$
\int_{\partial B \frac{1}{\gamma_{i}}}\|\nabla w\|^{2} \geq \frac{2}{r^{\frac{1}{\gamma_{i}}}} \int_{B_{r}^{\frac{1}{\gamma_{i}}}}\|\nabla w\|^{2}
$$

(The factor 2 comes from (3.2) with $\theta_{i+1}-\theta_{i}=\pi$.) We notice that by the construction of $w$ we have

$$
\int_{B_{r} \frac{1}{\gamma_{i}}}\|\nabla w\|^{2}=2 \int_{\sigma_{i}}\|\nabla \tilde{u}\|^{2}
$$


and

$$
\int_{\partial B \frac{1}{\gamma_{i}}}\left|w_{\tau}\right|^{2}=2 \gamma_{i} r^{1-\frac{1}{\gamma_{i}}} \int_{y_{i} \bar{y}_{i+1}}\left|\tilde{u}_{\tau}\right|^{2} .
$$

where $w_{\tau}$ (resp. $\tilde{u}_{\tau}$ ) is the tangential partial derivative on $\partial B_{r} \frac{1}{\gamma_{i}}$ (resp. on the associated arc $y_{i} \overparen{y_{i+1}}$ of $\left.\partial B_{r}(P)\right)$. From the construction of $\tilde{u}$ we know that $\tilde{u}_{\tau}=u_{\tau}$ on $\partial B_{r}(P)$. Identities (6.6), (6.7) and (6.8) gives then:

$$
\int_{\sigma_{i}}\|\nabla \tilde{u}\|^{2} \leq \gamma_{i} r \int_{y_{i} \hat{y}_{i+1}}\left|u_{\tau}\right|^{2} .
$$

Using (6.5) we deduce

$$
\int_{B_{r}(P) \backslash \tilde{K}}\|\nabla \tilde{u}\|^{2} \leq 2 \pi C_{1} r^{\beta} .
$$

Since $(u, K)$ is a minimizer of the Mumford-Shah functional we shall have

$$
J(u, K) \leq J(\tilde{u}, \tilde{K})
$$

which implies

$$
\begin{aligned}
\mathcal{H}^{1}\left(K \cap B_{r}(P)\right) & \leq \mathcal{H}^{1}\left(\tilde{K} \cap B_{r}(P)\right)+\int_{B_{r}(P) \backslash \tilde{K}}\|\nabla \tilde{u}\|^{2}+\int_{B_{r}(P)}(u-g)^{2} \\
& \leq 2 r+2 \pi C_{1} r^{\beta}+\pi r^{2}
\end{aligned}
$$

For any $R<R_{0}, \exists n \geq n_{0}$ such that $R \in\left[2^{-n-1}, 2^{-n}\right)$. We have proved that there is a $r \in\left[2^{-n}, 2^{-n+1}\right)$ such that (6.13) holds. The hypotheses of the lemma implies that for $R<r$,

$$
\mathcal{H}^{1}\left(K \cap B_{R}(P)\right)+2(r-R) \leq \mathcal{H}^{1}\left(K \cap B_{r}(P)\right)
$$

Therefore we have

$$
\mathcal{H}^{1}\left(K \cap B_{R}(P)\right) \leq 2 R+2 \pi C_{1} r^{\beta}+\pi r^{2}
$$

Let $C=2^{1+2 \beta} \pi C_{1}+16 \pi R_{0}^{2-\beta}$, since $r / 4<R<R_{0}$ and $\beta<2$ we get

$$
\forall R<R_{0}, \quad \mathcal{H}^{1}\left(K \cap B_{R}(P)\right) \leq 2 R+C R^{\beta} .
$$

Vol. 13, n 4-1996. 
PROPOSITION 6.4. - Under the assumptions of lemma 6.2 there is a constant $C\left(R_{0}, \beta\right)$ and a straight line $L$ passing through $P$ such that for all $R<R_{0} / 2$ :

$$
K \cap B_{R}(P) \subset\left\{X, d(X, L) \leq C R^{\frac{\beta+1}{2}}\right\}
$$

Proof. - Let $R$ be given, $R<R_{0} / 2$. Let $M$ and $N$ be two points of $K \cap \partial B_{R}(P)$ chosen in each connected component of $\partial B_{R}(P) \cap$ $\left\{X \in B_{R}(P), d\left(X, L_{R}\right)<l R\right\}$ (see lemma 6.2). If there is a point $X \in K \cap B_{R}(P)$ and a real $m \leq 2 l$ such that $d(X,[P, M]) \geq m R>0$ and $d(X,[P, N]) \geq m R>0$ then a straightforward estimation gives a constant $C$ such that $\mathcal{H}^{1}\left(K \cap B_{2 R}(P)\right) \geq 2 R+C R m^{2}$. We deduce from (6.3) that there is a constant $C$ such that $m \leq C R^{\frac{\beta-1}{2}}$. This is:

$$
\begin{aligned}
K \cap B_{R}(P) \subset & \left(\left\{X, d(X,[P, M]) \leq C R^{\frac{\beta+1}{2}}\right\}\right. \\
& \left.\cup\left\{X, d(X,[P, N]) \leq C R^{\frac{\beta+1}{2}}\right\}\right) .
\end{aligned}
$$

We can choose two sequences $M_{k}$ and $N_{k}$ of points in each connected component of $K \cap \partial B_{R / 2^{k}}(P)$. Identity (6.17) gives the estimates:

$$
d\left(M_{k+1},\left[P, M_{k}\right]\right) \leq C\left(R / 2^{k}\right)^{\frac{\beta+1}{2}} \text { and } d\left(N_{k+1},\left[P, N_{k}\right]\right) \leq C\left(R / 2^{k}\right)^{\frac{\beta+1}{2}} .
$$

Let $\theta_{k}$ (resp. $\Theta_{k}$ ) denotes a determination of the angle that the vector $P M_{k}$ (resp. $P N_{k}$ ) makes with a fixed vector $e$. Inequalities (6.18) implies that the determination of $\theta_{k+1}$ and $\Theta_{k+1}$ can be chosen such that:

$$
\left|\theta_{k+1}-\theta_{k}\right| \leq 2 C\left(R / 2^{k}\right)^{\frac{\beta-1}{2}} \text { and }\left|\Theta_{k+1}-\Theta_{k}\right| \leq 2 C\left(R / 2^{k}\right)^{\frac{\beta-1}{2}} .
$$

This gives the convergence of the sequences $\theta_{k}$ and $\Theta_{k}$ to real numbers $\theta_{\infty}$ and $\Theta_{\infty}$. We notice that since the blow-up limit at $P$ is a straight line we shall have $\theta_{\infty}=\Theta_{\infty}+(2 n+1) \pi$ where $n$ is an integer. This defines a line $L$ passing through $P$. Inequalities (6.19) give the existence of a constant $C:\left|\theta_{k}-\theta_{\infty}\right| \leq C\left(R / 2^{k}\right)^{\frac{\beta-1}{2}}$ and $\left|\Theta_{k}-\Theta_{\infty}\right| \leq C\left(R / 2^{k}\right)^{\frac{\beta-1}{2}}$. Together with (6.17) this gives the conclusion of the proposition.

Let $P$ be a flat point of $K$, we assume that there is an open neighborhood $\omega$ of $P$ such that $\omega$ intersects only one connected component of $K$. We will prove then that $K$ is $C^{1, \alpha}$ in a neighborhood of $P$.

LEMmA 6.5. - There is a neighborhood $U$ of $P$ such that $K \cap U$ is a Jordan arc and such that for all $l, 0<l<1$ there is a $R_{0}>0$ such that 
for all $M \in K \cap U, M$ is a flat point and for all $R<R_{0}$ there is a straight line $L_{R}^{M}$ passing through $M$ such that

$$
\left.\begin{array}{c}
K \cap B_{R}(M) \subset\left\{X \in B_{R}(M), d\left(X, L_{R}^{M}\right)<l R\right\}, \\
K \text { intersects each connected component } \\
\text { of } \partial B_{R}(M) \cap\left\{X \in B_{R}(M), d\left(X, L_{R}^{M}\right)<l R\right\} .
\end{array}\right\}
$$

Proof. - We first notice that proposition 5.3 gives the existence of a neighborhood of $P$ in which $K$ is a Jordan arc. In this neighborhood, any point of $K$ is a flat point. By a contradiction argument, if the conclusion of the lemma is not true then one can construct a sequence of flat points $M_{n}$ converging to a point $P$ and a sequence of positive real numbers $R_{n}$ converging to 0 such that (6.20) does not hold. By construction, the associated blow-up sequence $\left(u_{n}(X)=u\left(M_{n}+R_{n} X\right) / \sqrt{R_{n}}, K_{n}\right)$ cannot converge to a type (ii) global-minimizer. Since $K$ is an arc in a neighborhood of $P, \mathcal{H}^{1}\left(K \cap B_{R_{n}}\left(M_{n}\right)\right) \geq 2$ and the blow-up limit cannot be a type (iv) (neither (i)). Finally, a similar argument as in lemma 4.6 proves that the blow-up limit cannot be of type (iii) (triple-point). This contradicts theorems 2.2 and 4.1.

Proof of theorem 6.1. - From lemma 6.5 we know that the hypotheses of lemma 6.2 and 6.3 are satisfied uniformly in a neighborhood of $P$. Then the conclusion of proposition 6.4 holds uniformly in this neighborhood. This gives exactly the $C^{1, \alpha}$ regularity for $\alpha=\frac{\beta-1}{2}$. Since this is true for any $l, 0<l<\frac{\sqrt{3}}{2}$ and since $\beta(l) \rightarrow 2$ when $l \rightarrow 0$ we have proved the $C^{1, \alpha}$ regularity for any $\alpha<1 / 2$.

Notice that the same arguments as in lemma 6.2 in the case of a triple point $P$ give the

LeMma 6.6. - Let $P$ be a triple point of $K$, such that there is an open neighborhood $\omega$ of $P$ such that $\omega$ intersects only one connected component of $K$. Then for any $\beta<3$ there is a constant $C$ such that

$$
\phi_{P}(R) \leq C R^{\beta} \text {. }
$$

Then, the procedure of lemma 6.3 and proposition 6.4 yields

Proposition 6.7. - Under the conditions of lemma 6.6, there are three half lines $D_{i}$ meeting at $P$ with angle $2 \pi / 3$ such that for any $\beta<3$ there is a constant $C$ such that

$$
K \cap B_{R} \subset\left\{X, d\left(X, D_{1} \cup D_{2} \cup D_{3}\right) \leq C R^{\frac{\beta+1}{2}}\right\} .
$$

Taking this into account in the proof of theorem 6.1 we write the: 
THEOREM 6.8. - Let $P$ be a triple point of $K$, such that there is an open neighborhood $\omega$ of $P$ such that $\omega$ intersects only one connected component of $K$. Then there is a neighborhood $U$ of $P$ in which $K$ is the union of three $C^{1, \alpha}$ arcs $(\forall \alpha<1 / 2)$ making a $2 \pi / 3$ angle at $P$.

COROLlary 6.9. - If $G$ is an isolated connected component of $K$ then it is the union of a finite number of arcs. Those arcs are $C^{1, \alpha}$ away from the crack-tips and can only merge through a finite number of triple points.

\section{FURTHER REGULARITY}

\section{1. $C^{1,1}$ regularity}

In this section we will prove in theorem 7.2 that if $K$ is, locally, a $C^{1, \alpha}$ curve then it is $C^{1,1}$. This result together with theorem 5.1 and 6.1 completes the proof of theorem 1.1 and 1.2. We first start with a proposition which gives the $C^{1, \alpha}$ regularity of $u$ up to the edge set $K$. In the following, $(u, K)$ is a minimizer of the Mumford-Shah functional.

Proposition 7.1. - Let $P$ be a point of $K$ such that in a neighborhood $B_{4 R}(P)$ of $P$ the edge set $K$ is a $C^{1, \alpha}$ curve: $\{(x, y), y=f(x), x \in I\} . K$ defines two sets $B_{R}^{+}=\left\{(x, y) \in B_{R}(P), y \geq f(x)\right\}$ and $B_{R}^{-}=\{(x, y) \in$ $\left.B_{R}(P), y \leq f(x)\right\}$. The minimizer $u$ can be extended on each side of $K$ such that

$$
u \in C^{1, \alpha}\left(B_{R}^{+}\right) \quad \text { and } \quad u \in C^{1, \alpha}\left(B_{R}^{-}\right) .
$$

Proof. - We will prove that $u$ is $C^{1, \alpha}$ in $B_{R}^{+}=\left\{(x, y) \in B_{R}(P), y \geq\right.$ $f(x)\}$. The minimizer $u$ satisfies $-\Delta u+u-g=0$ in the interior of $B_{4 R}^{+}$. In the sense of lemma 3.1 in [6] we can say that $u$ satisfies Neumann boundary conditions $\frac{\partial u}{\partial \nu}=0$ on $K$. We want to prove that a solution of $-\Delta u+u-g=0$ in $B_{4 R}^{+}$with $\frac{\partial u}{\partial \nu}=0$ on $K$ is $C^{1, \alpha}$ in $B_{R}^{+}$. Since $g$ is $L^{\infty}$, the $C^{1, \alpha}$ regularity in the case of Dirichlet conditions is given by theorem 5.5.5' of [18]. Following [18], it is possible in fact to prove the $C^{1, \alpha}$ regularity in the case of Neumann boundary conditions. For sake of completeness the proof is given in the appendix.

THEOREM 7.2. - Let $P$ be a point of $K$ such that in a neighborhood $U$ of $P$ the edge set $K$ is a $C^{1, \alpha}$ curve, then $K$ is $C^{1,1}$ in $U$.

Proof. - For every point $M$ of $K \cap U$ we may take a ball $B_{r}(M) \subset U$ and a coordinate system centered at $M$ in which $K \cap B_{r}(M)$ is the graph 
of a $C^{1, \alpha}$ function $f: I \rightarrow \mathbf{R}$ such that $f(0)=0$ and $f^{\prime}(0)=0$ :

$$
K \cap B_{r}(M)=\{(x, y) \in U, x \in I \text { and } y=f(x)\}
$$

We would like to estimate if we can reduce the Mumford-Shah energy by moving the edge set near $M$. This can be achieve for instance by adding a $C_{0}^{\infty}(I)$ function $\zeta$ to $f$. Assume that $\zeta \geq 0$, let us replace $K$ in $B_{r}(M)$ by the set $\left\{(x, y) \in B_{r}(M), y=f(x)+\zeta(x)\right\}$ and get a new edge set $\tilde{K}$. We define now a new function $\tilde{u}$ from $u$ in $B_{r}(M) \backslash \tilde{K}$ :

$$
\begin{aligned}
\forall(x, y) \in B_{r}(M), & \text { if } f(x)<y<f(x)+\zeta(x) \\
& \text { then } \tilde{u}(x, y)=u(x, 2 f(x)-y), \\
& \text { if not, then } \tilde{u}(x, y)=u(x, y) .
\end{aligned}
$$

It is easy to compute:

$$
\begin{aligned}
J(\tilde{u}, \tilde{K})-J(u, K)= & \int_{I}\left(\sqrt{1+\left(f^{\prime}+\zeta^{\prime}\right)^{2}}-\sqrt{1+\left(f^{\prime}\right)^{2}}\right) d x \\
& +\int_{\{(x, y), f(x)<y<f(x)+\zeta(x)\}}\left((\tilde{u}-g)^{2}-(u-g)^{2}\right. \\
& \left.+\|\nabla \tilde{u}\|^{2}-\|\nabla u\|^{2}\right) d x d y .
\end{aligned}
$$

Since $(u, K)$ is a minimizer we must have $J(\tilde{u}, \tilde{K})-J(u, K) \geq 0$ that is:

$$
\begin{aligned}
& \int_{I}\left(\sqrt{1+\left(f^{\prime}+\zeta^{\prime}\right)^{2}}-\sqrt{1+\left(f^{\prime}\right)^{2}}\right) d x \\
& \quad+\int_{\{(x, y), f(x)<y<f(x)+\zeta(x)\}}\left((\tilde{u}-g)^{2}-(u-g)^{2}\right. \\
& \left.\quad+\|\nabla \tilde{u}\|^{2}-\|\nabla u\|^{2}\right) d x d y \geq 0 .
\end{aligned}
$$

We may replace $\zeta$ by $t \zeta$ and let $t>0$ go to 0 . We get then:

$$
\frac{1}{t} \int_{I}\left(\sqrt{1+\left(f^{\prime}+\zeta^{\prime}\right)^{2}}-\sqrt{1+\left(f^{\prime}\right)^{2}}\right) \rightarrow \int_{I} \frac{f^{\prime} \zeta^{\prime}}{\sqrt{1+\left(f^{\prime}\right)^{2}}}
$$

We denote by $\kappa$ the distributional first order derivative of $\frac{f^{\prime}}{\sqrt{1+\left(f^{\prime}\right)^{2}}}$. It is the curvature of $K$ and we can write:

$$
\int_{I} \frac{f^{\prime} \zeta^{\prime}}{\left(1+\left(f^{\prime}\right)^{2}\right)^{1 / 2}}=-\int_{I} \kappa \zeta
$$

Vol. 13, $\mathrm{n}^{\circ} 4-1996$. 
Since $u$ and $f$ are $C^{1, \alpha}$ and since $g$ is bounded we deduce from (7.3) that there is a constant $C$ such that:

$$
\int_{I} \kappa \zeta \leq C \int_{I} \zeta
$$

A similar construction with $-\zeta$ gives the existence of a constant $C$ such that for all $C_{0}^{\infty}(I)$ function $\theta$ :

$$
\left|\int_{I} \kappa \theta\right| \leq C \int_{I}|\theta|
$$

This means that $\kappa$ (and therefore $f^{\prime \prime}$ ) is an $L^{\infty}$ function and that $K \cap B_{r}(M)$ is a $C^{1,1}$ curve. This is true for every point $M \in K \cap U$. The proof of theorem 7.2 is completed.

\section{2. $C^{k+2, \alpha}$ regularity when $g$ is $C^{k, \alpha}$}

In this section we prove theorem 1.4. From theorem 6.1, we know that if $K$ is a $C^{1}$ curve then it is $C^{1, \alpha}$. We follow then the same construction as in the proof of theorem 7.2. With the same notations, we start over from inequality (7.3). We denote by $u^{+}(x), \nabla u^{+}(x), \tilde{u}^{+}(x)$ and $\nabla \tilde{u}^{+}(x)$ the limit value of $u, \nabla u, \tilde{u}$ and $\nabla \tilde{u}$ on $K$ from above at the point $(x, f(x))$. We write (7.3) with $t \zeta$ in place of $\zeta$. We notice that the continuity of $g$ allows to rewrite (7.3) in the limit $t \rightarrow 0$ as:

$$
\begin{aligned}
& \int_{I} \zeta\left(-\frac{f^{\prime \prime}}{\left(1+\left(f^{\prime}\right)^{2}\right)^{3 / 2}}+\left(\tilde{u}^{+}-g(x, f(x))\right)^{2}\right. \\
& \left.\quad-\left(u^{+}-g(x, f(x))\right)^{2}+\left\|\nabla \tilde{u}^{+}\right\|^{2}-\left\|\nabla u^{+}\right\|^{2}\right) d x \geq 0
\end{aligned}
$$

We denote by $u^{-}(x)$ and $\nabla u^{-}(x)$ the limit value of $u$ and $\nabla u$ on $K$ from below at the point $(x, f(x))$. Inequality (7.8) gives at almost every $x \in I$ :

$$
\begin{aligned}
& -\frac{f^{\prime \prime}(x)}{\left(1+\left(f^{\prime}(x)\right)^{2}\right)^{3 / 2}}+\left(u^{-}(x)-g(x, f(x))\right)^{2}-\left(u^{+}(x)-g(x, f(x))^{2}\right. \\
& +\left\|\nabla u^{-}(x)\right\|^{2}-\left\|\nabla u^{+}(x)\right\|^{2} \geq-4\left\|\nabla u^{-}(x)\right\|^{2}\left(\left|f^{\prime}(x)\right|+\left(f^{\prime}(x)\right)^{2}\right)(7.9)
\end{aligned}
$$

With $-\zeta$ we derive at almost every $x \in I$ :

$$
\begin{aligned}
& -\frac{f^{\prime \prime}(x)}{\left(1+\left(f^{\prime}(x)\right)^{2}\right)^{3 / 2}}+\left(u^{-}(x)-g(x, f(x))\right)^{2}-\left(u^{+}(x)-g(x, f(x))^{2}\right. \\
& +\left\|\nabla u^{-}(x)\right\|^{2}-\left\|\nabla u^{+}(x)\right\|^{2} \leq 4\left\|\nabla u^{+}(x)\right\|^{2}\left(\left|f^{\prime}(x)\right|+\left(f^{\prime}(x)\right)^{2}\right)
\end{aligned}
$$


Since $f^{\prime}(0)=0$, there exists a function $\varepsilon_{0}$ with $\lim _{0} \varepsilon_{0}(x)=0$ such that for almost every $x \in I$ :

$$
\begin{aligned}
& \mid-\frac{f^{\prime \prime}(x)}{\left(1+\left(f^{\prime}(x)\right)^{2}\right)^{3 / 2}}+\left(u^{-}(x)-g(x, f(x))\right)^{2}-\left(u^{+}(x)-g(x, f(x))^{2}\right. \\
& +\left\|\nabla u^{-}(x)\right\|^{2}-\left\|\nabla u^{+}(x)\right\|^{2} \mid \leq \varepsilon_{0}(x)
\end{aligned}
$$

This inequality has been derived by an argument centered at $M$ of coordinates $(0,0)$. The same argument at a point $N\left(x_{0}, f\left(x_{0}\right)\right)$ would give the existence of a function $\varepsilon_{x_{0}}$ with $\lim _{x_{0}} \varepsilon_{x_{0}}(x)=0$ such that for almost every $x \in I$ :

$$
\begin{aligned}
& \mid-\frac{f^{\prime \prime}(x)}{\left(1+\left(f^{\prime}(x)\right)^{2}\right)^{3 / 2}}+\left(u^{-}(x)-g(x, f(x))\right)^{2} \\
& -\left(u^{+}(x)-g(x, f(x))^{2}\right. \\
& +\left\|\nabla u^{-}(x)\right\|^{2}-\left\|\nabla u^{+}(x)\right\|^{2} \mid \leq \varepsilon_{x_{0}}(x)
\end{aligned}
$$

Consequently $f^{\prime \prime}$ is continuous and at every point $N(x, f(x))$ of $K \cap B_{r}(M)$ we have the expression of the curvature of $K$ at the point $N$ :

$$
\begin{aligned}
& \frac{f^{\prime \prime}(x)}{\left(1+\left(f^{\prime}(x)\right)^{2}\right)^{3 / 2}}=\left(u^{-}(x)-g(N)\right)^{2}-\left(u^{+}(x)-g(N)\right)^{2} \\
& +\left\|\nabla u^{-}(x)\right\|^{2}-\left\|\nabla u^{+}(x)\right\|^{2}
\end{aligned}
$$

From this equation we are able to start a classical bootstrap argument. If $g$ is $C^{0, \alpha}$ then $f$ is $C^{2, \alpha}$ and then (elliptic regularity) $u$ is $C^{2, \alpha}$ up to $K$.

By induction, if $g$ is $C^{k, \alpha}$ and $u$ is $C^{k+1, \alpha}$ up to $K$ then $f$ is $C^{k+2, \alpha}$ and $u$ is $C^{k+2, \alpha}$ up to $K$. This completes the proof of theorem 1.4.

Remark 7.3. - Notice (7.14) makes it easy to construct an example of an $L^{\infty}$ function $g$ such that $K$ is $C^{1,1}$ and not $C^{2}$.

\subsection{Minimization with $n$ connected components}

One can study the problem: minimize the Mumford-Shah functional under the condition that $K$ is made at most of $n$ connected components 
(see proposition 15.44 of [17]). The previous analysis gives immediately the proof of theorem 1.5 .

Remark 7.4. - The proof through blow-up procedure (theorem 2.3) gives that the maximum number of arcs and triple points do not depend on the image $g, 0 \leq g \leq 1$.

\section{REGULARITY ALMOST EVERYWHERE}

In this section we will not make any assumption on the connected components of $K$ and prove theorem 1.3. This result is a direct consequence of the following proposition and of theorem 6.1 and 7.2. The proof of proposition 8.1 involves an argument of coarea formula as in [7]. The coarea formula is used, here, together with blow-up techniques and precise estimates on the length of the level set of a distance function.

Proposition 8.1. - For $\mathcal{H}^{1}$ almost every point $P$ of $K$ there is a ball $B_{\rho}(P)$ and a one-to-one Lipschitz map $\psi:[0,1] \rightarrow \Omega$ such that

$$
P \in \psi((0,1)) \quad \text { and } \quad B_{\rho}(P) \cap K=B_{\rho}(P) \cap \psi([0,1]) .
$$

Proof. - $\mathcal{H}^{1}$ almost every point of $K$ has a tangent and has density one. Therefore by a blow-up at such points one gets a global minimizer $\left(u_{0}, K_{0}\right)$ where $K_{0}$ is included in a line (the tangent) and where $\mathcal{H}^{1}\left(K_{0} \cap B_{r}\right)=2 r$ for all $r$. Then, $K_{0}$ is a line and $u_{0}$ is a constant on both sides. Let $P$ be such a point of $K$ and $\left(u_{\varepsilon}, K_{\varepsilon}\right)$ be a blow-up sequence at $P$.

Step 1. - There are no holes in $K_{\varepsilon}$ near $P$.

Assume that $K_{0}$ coincides with the $x$ axis. For $\varepsilon$ small, $\left(u_{\varepsilon}, K_{\varepsilon}\right)$ is arbitrary close to $\left(u_{0}, K_{0}\right)$ and for any $A>0$ there is $\varepsilon>0$ such that $\left(U_{\varepsilon}(X)=u(P+\varepsilon X) / \sqrt{\varepsilon}-\right.$ Const, $\left.K_{\varepsilon}\right)$ satisfies in the rectangle $R_{2}=[-2,2] \times[-1,1]$ :

$$
\left.\begin{array}{l}
U_{\varepsilon}(x, y) \geq 2 A, \quad \text { if } x \geq 1 / 2, \quad(x, y) \in R_{2}, \\
U_{\varepsilon}(x, y) \leq-2 A, \quad \text { if } x \leq-1 / 2, \quad(x, y) \in R_{2}, \\
K_{\varepsilon} \cap R_{2} \subset\{-1 / 2<x<1 / 2\}, \\
\left|\mathcal{H}^{1}\left(K_{\varepsilon}\right)-2\right| \leq 1 / A, \mathbf{d}\left(K_{\varepsilon}, K_{0}\right) \leq 1 / A, \\
\int_{R_{2} \backslash K_{\varepsilon}}\left\|\nabla U_{\varepsilon}\right\|^{2} \leq 1 / A .
\end{array}\right\}
$$


Let $x_{0} \in[-2,2]$ be such that $K_{\varepsilon}$ intersects the vertical segment $\left\{x_{0}\right\} \times[-1,1]$ in a single point $\left(x_{0}, y_{0}\right)$. Assume that

$\exists y \in\left(y_{0}, 1 / 2\right), \quad U_{\varepsilon}\left(x_{0}, y_{0}\right)<A$ or $\exists y \in\left(-1 / 2, y_{0}\right), \quad U_{\varepsilon}\left(x_{0}, y_{0}\right)>-A$

then $\int_{-1}^{1}\left\|\nabla U_{\varepsilon}\right\|^{2} d y \geq A^{2}$. For large $A, \int_{R_{2} \backslash K_{\varepsilon}}\left\|\nabla U_{\varepsilon}\right\|^{2}$ is small and there is at least a $x_{0} \in[1,2]$ such that $K_{\varepsilon}$ intersects $\left\{x_{0}\right\} \times[-1,1]$ and $\left\{-x_{0}\right\} \times[-1,1]$ in a single point and such that (8.3) does not occur. Replace $\varepsilon$ by $\varepsilon x_{0}$ and let $R_{1}=[-1,1] \times[-1,1]$. The set $K_{\varepsilon}$ intersects $\partial R_{1}$ at two points $M_{1}=\left(-1, y_{1}\right)$ and $M_{2}=\left(1, y_{2}\right)$ and divides $\partial R_{1} \backslash K_{\varepsilon}$ in two parts: the upper one where $U_{\varepsilon}>A$ and the lower one where $U_{\varepsilon}<-A$. Moreover, for $\varepsilon$ small, properties (8.2) are still satisfied in $R_{1}$.

We will now prove that for $A$ large, there are no holes in $K_{\varepsilon}$, i.e. $M_{1}$ and $M_{2}$ are in the same connected component of $K_{\varepsilon} \cap R_{1}$. Consider a path $\psi:[0, l] \rightarrow R_{2}$ connecting $M_{1}$ to $M_{2}$ and such that $\|\nabla \psi\|=1$ a.e. and such that $\gamma=\mathcal{H}^{1}\left(\psi[0,1] \backslash K_{\varepsilon}\right)$ is minimal. Assume by contradiction that $\gamma \neq 0$ (there are holes in $\left.K_{\varepsilon}\right)$. We want to prove that taking $\tilde{K}=K_{\varepsilon} \cup \psi([0, l])$ instead of $K$ we reduce the energy $\int_{\left\{R_{1} \backslash K_{\varepsilon}\right\}}\left\|\nabla U_{\varepsilon}\right\|^{2}$ by more than $\gamma$ and contradict the minimality of $\left(U_{\varepsilon}, K_{\varepsilon}\right)$.

For this purpose we notice that if:

$$
\begin{aligned}
& \phi:[0, a] \rightarrow \mathbf{R}_{1} \backslash K_{\varepsilon} \text { is a one-to-one path such that } \\
& \|\nabla \phi\|=1 \text { a.e., } \phi(0) \text { and } \phi(a) \in \partial R_{1}, \\
& U_{\varepsilon}(\phi(0)) \leq-A \text { and } U_{\varepsilon}(\phi(0)) \geq A
\end{aligned}
$$

then $\int_{0}^{a}\left\|\nabla U_{\varepsilon}(\phi(t))\right\|^{2} d t \geq 4 A^{2} / a$. We will then construct a family of such curves as part of the level surfaces of a distance function.

Assume that $K_{\varepsilon} \cap R_{1}$ is made of a finite number of pieces and that the minimum path $\psi$ touches successively the components $F_{0} \ni M_{1}$, $F_{1}, \ldots, F_{n} \ni M_{2}$. We construct the dilation of $F_{0}$. For $\delta$ small, $R_{1} \cap \partial\left\{N \in \mathbf{R}^{2}, d\left(N, F_{0}\right) \leq \delta\right\}$ does not intersects $K_{\varepsilon}$ and one component of this boundary is a curve $\phi_{\delta}$ satisfying (8.4), such that its length $l_{\delta}$ is less than $2 \mathcal{H}^{1}\left(F_{0}\right)+\pi \delta$. We proceed until $\phi_{\delta}\left(\left[0, l_{\delta}\right]\right)$ touches $K_{\varepsilon}$ for $\delta=\delta_{0}$, then we replace $F_{0}$ by the union $G_{1}$ of $\left\{N \in R_{1}, d\left(N, F_{0}\right) \leq \delta_{0}\right\}$ and the connected components of $K_{\varepsilon}$ touched by $\phi_{\delta_{0}}\left(\left[0, l_{\delta_{0}}\right]\right)$. We proceed as before: for $\delta>\delta_{0}, \phi_{\delta}$ is the component satisfying (8.4) of the set $R_{1} \cap \partial\left\{N \in \mathbf{R}^{2}, d\left(N, G_{1}\right) \leq \delta-\delta_{0}\right\}$. We proceed until $\phi_{\delta}\left(\left[0, l_{\delta}\right]\right)$ touches $K_{\varepsilon}$, etc. We remark that by construction, $\phi_{\delta}$ intersects $\psi([0, l])$ at a point $\psi(\tau)$ such that $\mathcal{H}^{1}\left(\psi([0, \tau]) \backslash K_{\varepsilon}\right)=\delta$. And if $\psi(\tau) \notin K_{\varepsilon}$ then, $\phi_{\delta}\left(\left[0, l_{\delta}\right]\right)$ 
cannot intersect any of the $F_{i}$, or this would contradict the minimality of $\psi$. Consequently, the construction can be done for any $\delta \in[0, \gamma]$. For all $\delta$, except a finite number of them, $\phi_{\delta}$ satisfies condition (8.4) with a length $l_{\delta}$ less than $2 \mathcal{H}^{1}\left(K_{\varepsilon} \cap R_{1}\right)+\pi \delta$. Moreover, we have $d\left(\phi_{\delta}(t), \phi_{\delta^{\prime}}\left(t^{\prime}\right)\right) \geq\left|\delta-\delta^{\prime}\right|$ for all $\delta, \delta^{\prime}$ and $t \in\left[0, l_{\delta}\right], t^{\prime} \in\left[0, l_{\delta^{\prime}}\right]$. With this construction, the curves $\phi_{\delta}$ can be viewed as part of the level surfaces of the distance function $D$ where $D(X)$ is the infimum of $\mathcal{H}^{1}\left(\xi([0,1]) \backslash K_{\varepsilon}\right)$ with $\xi(0)=M_{1}$ and $\xi(1)=X$. On each curve $\phi_{\delta}, \int_{\phi_{\delta}}\left\|\nabla U_{\varepsilon}\right\|^{2}$ is larger than $4 A^{2} /\left(2 \mathcal{H}^{1}\left(K_{\varepsilon} \cap R_{1}\right)+\pi \gamma\right)$. The coarea formula [12] gives

$$
\int_{R_{1} \backslash K_{\varepsilon}}\left\|\nabla U_{\varepsilon}\right\|^{2} \geq\left(\frac{4 A^{2}}{2 \mathcal{H}^{1}\left(K_{\varepsilon} \cap R_{1}\right)+\pi \gamma}\right) \gamma
$$

In general, we take the sequence $\left(F_{n}\right)_{n \in \mathbf{N}}$ of components of $K_{\varepsilon} \cap R_{1}$ touched by $\psi$ and $\left(G_{n}\right)_{n \in \mathrm{N}}$ the other components of $K_{\varepsilon} \cap R_{1}$. We first remove the $G_{n}$. Then we choose a finite number of holes of total length larger than $\gamma / 2$ (i.e. a finite number of disjoint intervals $\left(a_{k}, b_{k}\right)$ of total length larger than $\gamma / 2$ in $\psi^{-1}\left(\psi\left([0, l] \backslash K_{\varepsilon}\right)\right)$ and fill in the other holes with the appropriate pieces of $\psi([0, l])$ (i.e. we take $\left.K^{\prime}=\cup F_{n} \cup \psi\left([0, l] \backslash \cup\left(a_{k}, b_{k}\right)\right)\right)$. This way, we are reduced, with $K^{\prime}$, to the previous case with a finite number of pieces and may construct the curves $\phi_{\delta}$ for $\delta \in[0, \gamma / 2]$. We introduce the one-to-one function $h_{0}(t, \delta)=\phi_{\delta}(t)$ for $t \in\left[0, l_{\delta}\right]$. We may introduce successively the $G_{n}$ and construct a function $h_{n}$ ( $h_{n}$ is a small perturbation of $\left.h_{n-1}\right)$. The sequence $h_{n}$ converges to a function $h$ such that for almost every $\delta$, $t \mapsto h(t, \delta)$ satisfies (8.4), has a length less than $2 \mathcal{H}^{1}\left(K_{\varepsilon} \cap R_{1}\right)+\gamma+\pi \delta$ and $d\left(h(t, \delta), h\left(t^{\prime}, \delta^{\prime}\right)\right) \geq\left|\delta-\delta^{\prime}\right|$. Again, the coarea formula gives $\int_{R_{1} \backslash K_{\varepsilon}}\left\|\nabla U_{\varepsilon}\right\|^{2} \geq C A^{2} \gamma$ where $C=2 /\left(2 \mathcal{H}^{1}\left(K_{\varepsilon} \cap R_{1}\right)+(\pi+1) \gamma\right)$.

Now, if we replace $K_{\varepsilon}$ by $\tilde{K}=K_{\varepsilon} \cup \psi([0,1])$, then $\mathcal{H}^{1}(\tilde{K})=\mathcal{H}^{1}(K)+\gamma$ and $\tilde{K}$ cut $R_{1}$ in two parts. In the upper part, take $\tilde{U}=\sup \left(A, U_{\varepsilon}\right)$ and in the lower part take $\tilde{U}=\inf \left(-A, U_{\varepsilon}\right)$. For almost every curve $t \mapsto h(t, \delta)$ there is a minimum segment $[\alpha, \beta]$ in which $t \mapsto U_{\varepsilon}(h(t, \delta))$ goes from $-A$ to $A$ and one gets as above $\int_{\left\{-A \leq U_{\varepsilon} \leq A\right\} \backslash K_{\varepsilon}}\left\|\nabla U_{\varepsilon}\right\|^{2} \geq C A^{2} \gamma$ which is larger than $\gamma$ for large $A$. Therefore, $(\tilde{U}, \tilde{K})$ is a compact perturbation of $\left(U_{\varepsilon}, K_{\varepsilon}\right)$ which reduces the energy. This contradicts the minimality of $\left(U_{\varepsilon}, K_{\varepsilon}\right)$.

Consequently, for $\varepsilon$ small, $M_{1}$ and $M_{2}$ are in the same connected component of $K_{\varepsilon}$

Step 2. - The blow-up limit at any point near $P$ is a line. 
Let $P$ be a point where $K$ has a tangent and has density one. Then, for any $\beta, \beta^{\prime}, \exists r_{0}$ such that $\forall r \leq r_{0}$

(a) the set $G_{r}=\left\{\rho \in[r / 2, r), \#\left(K \cap \partial B_{\rho}(P)\right)=1\right\}$ satisfies meas $\left(G_{r}\right)>(1-\beta) r / 2$,

(b) $\forall \rho \in G_{r} ; K \cap \partial B_{\rho}(P)$ is made of two points of the same connected component of $K$ (consequence of step 1) and of polar coordinates centered at $P,\left(\rho, \theta_{1}\right),\left(\rho, \theta_{2}\right)$ such that $\pi \beta^{\prime}>\left|\theta_{2}-\theta_{1}-\pi\right|$.

Those two properties hold then for the blow-up $K_{\varepsilon}, \forall r \leq r_{0} / \varepsilon$. The convergence of $K_{\varepsilon}$ to the line $K_{0}$ gives the existence of $r_{1}$ and $\varepsilon_{1}$ such that $\forall \varepsilon<\varepsilon_{1}, \forall Q \in K_{\varepsilon} \cap B_{1}(O), \forall r, 1 \leq r \leq r_{1} / \varepsilon_{1}$ properties (a) and (b) above holds at $Q$. Assume that there are sequences $\varepsilon_{n} \rightarrow 0, Q_{n} \in K_{\varepsilon_{n}} \cap B_{1}$ and $r_{n}, 0<r_{n}<1$ such that (a) and (b) are satisfied for $r, r_{n}<r<r_{1} / \varepsilon_{n}$ but not at $r=r_{n}$. Consider the blow-up sequence $V_{n}(X)=\frac{U_{\varepsilon_{n}}\left(Q_{n}+r_{n} X\right)}{\sqrt{r_{n}}}$, $v_{n}(X)=V_{n}(X)-c_{n}(X)$ (where $c_{n}$ is piecewise constant as in section 2), $K_{n}=\left\{X, Q_{n}+r_{n} X \in K_{\varepsilon_{n}}\right\}$. A subsequence will converge to a global minimizer $\left(v, K_{v}\right)$ such that $O \in K_{v}$, identities (a) and (b) (with $\geq$ instead of $>$ ) are satisfied for $r>1$ and

$$
\text { meas }\left(G_{1}\right) \leq(1-\beta) 1 / 2 \text { or } \exists \rho \in G_{1} \text { such that } \pi \beta^{\prime} \leq\left|\theta_{2}-\theta_{1}-\pi\right| \text {. }
$$

For $r>2$, the computation for the monotonicity formula gives for all $\rho \in G_{r}$

and then

$$
\int_{\partial B_{\rho} \backslash K_{v}}\|\nabla v\|^{2} \geq \frac{1}{\rho} \frac{2}{(1-\beta)} \int_{B_{\rho} \backslash K_{v}}\|\nabla v\|^{2}
$$

$$
\int_{B_{r / 2} \backslash K_{v}}\|\nabla v\|^{2} \leq 2^{-2+\tau} \int_{B_{r} \backslash K_{v}}\|\nabla v\|^{2},
$$

where $\tau\left(\beta, \beta^{\prime}\right) \rightarrow 0$ when $\beta, \beta^{\prime}$ goes to 0 . Assume that $\beta, \beta^{\prime}$ were chosen such that $\tau<1$. Then, since $\int_{B_{r} \backslash K_{v}}\|\nabla v\|^{2} \leq 2 \pi r$ one gets for all $r \geq 1$, and $n \geq 1, \int_{B_{r} \backslash K_{v}}\|\nabla v\|^{2} \leq 2^{n(-2+\tau)} 2 \pi r 2^{n}=2 \pi r 2^{n(-1+\tau)}$. Thus, $\int_{B_{r} \backslash K_{v}}\|\nabla v\|^{2}=0$ for all $r$ and the global minimizer $\left(v, K_{v}\right)$ is of type (i), (ii) or (iii) of theorem 4.1. Only (ii) satisfies (a) and (b) above. Therefore $K_{v}$ is a line, which contradicts (8.6). Consequently, (a) and (b) holds for all $r<r_{1}$ at any point $Q \in K \cap B_{\varepsilon_{1}}$. The blow-up at $Q$ is then a line.

\section{Step 3. - Conclusion.}

We have proved that there is a ball $B_{\sigma}$ such that $\partial B_{\sigma} \cap K=\left\{N_{0}, N_{1}\right\}$ and there is a Jordan arc $\psi:[0, l] \rightarrow \bar{B}_{\sigma} \cap K$ with endpoints $N_{0}$ and $N_{1}$. We know also that for any $Q \in B_{\sigma} \cap K$ the blow-up limit is a line. As in section 5 we conclude then that $\bar{B}_{\sigma} \cap K=\psi[0, l]$ which completes the proof of the proposition. 


\section{COLOR SEGMENTATION}

In the previous sections we have concentrate on black-and-white images. Mumford-Shah segmentation procedure can also be applied to color images. Following [10] we say that each point of a color image is characterized by its red, green and blue intensity. The true image is then the vector function $g: \Omega \rightarrow \mathbf{R}^{3}$ of red, green and blue intensity. We may assume that $g$ (and therefore the regularized image $u$ ) takes its values in the cube $[0,1]^{3}$ on which we define a Riemannian metric:

$$
(d s)^{2}=\sum_{i, j} \phi_{i j}(u) d u^{i} d u^{j}
$$

where $\phi_{i j}$ are continuous functions. We assume that there are two positive constants, $\lambda$ and $\mu$ such that

$$
\forall u_{0} \in[0,1]^{3}, \quad \forall x \in \mathbf{R}^{3}, \quad \lambda\|x\|^{2} \leq \sum_{i, j} \phi_{i j}\left(u_{0}\right) x^{i} x^{j} \leq \mu\|x\|^{2} .
$$

Mumford-Shah functional is then replaced by

$$
E(u, K)=\int_{\Omega \backslash K} d(u(x), g(x))+\int_{\Omega \backslash K} \sum_{i, j} \phi_{i j}(u) \nabla u^{i} \cdot \nabla u^{j}+\mathcal{H}^{1}(K) .
$$

The distance $d(u(x), g(x))$ is classically defined as the infimum

$$
d(u(x), g(x))=\inf _{v}\left(\int_{0}^{1} \sqrt{\sum_{i, j} \phi_{i j} \frac{d v^{i}}{d t} \frac{d v^{j}}{d t}} d t\right)
$$

over paths $v$ connecting $u(x)$ to $g(x)$ (a path is a smooth function $v:[0,1] \rightarrow[0,1]^{3}$ such that $v(0)=u(x)$ and $\left.v(1)=g(x)\right)$.

We may study, as in section 2, blow-up sequences $\left(u_{\varepsilon}, K_{\varepsilon}\right)$. Condition (9.2) ensures the convergence of a blow-up sequence to a blow-up limit $\left(u_{0}, K_{0}\right)$. Since $\phi_{i j}$ is continuous, we may choose a subsequence such that in each connected component of $\mathbf{R}^{2} \backslash K_{0}, \phi_{i j}\left(u_{\varepsilon}\right)$ converges locally uniformly to a constant. Since $K_{0}$ is a closed set there is a countable family of connected component $\mathcal{C}^{n}$. For each $\mathcal{C}^{n}$ we denote by $a_{i j}^{n}$ the limit value of $\phi_{i j}\left(u_{\varepsilon}\right)$ in $\mathcal{C}^{n}$. The argument of the proof of theorem 2.2 gives then

Proposition 9.1. - For any bounded open sub-domains $U, V$ of $\mathbf{R}^{2}$ with $U \subset \subset V$ there is no function $\tilde{u}_{0}$ and closed edge set $\tilde{K}_{0}$ defined on $V$ 
such that

(i) $\tilde{u}_{0}$ and $u_{0}$ (resp. $\tilde{K}_{0}$ and $K_{0}$ ) coincide in $V \backslash \bar{U}$,

(ii) for every pair of points $X, Y$ in $V \backslash\left(\bar{U} \cup K_{0}\right)$, if $X$ and $Y$ are in two distinct connected components of $V \backslash K_{0}$ so they are in $V \backslash \tilde{K}$,

(iii) $\left(\tilde{u}_{0}, \tilde{K}_{0}\right)$ lowers the energy

$$
\begin{aligned}
& \sum_{m} \int_{\tilde{\mathcal{C}}^{m}} \sum_{i, j} \tilde{a}_{i j}^{m} \nabla \tilde{u}_{0}^{i} \cdot \nabla \tilde{u}_{0}^{j}+\mathcal{H}^{1}(\tilde{K} \cap V) \\
& \leq \sum_{n} \int_{\mathcal{C}^{n} \cap V} \sum_{i, j} a_{i j}^{n} \nabla u_{0}^{i} \cdot \nabla u_{0}^{j}+\mathcal{H}^{1}(K \cap V),
\end{aligned}
$$

where $\tilde{\mathcal{C}}^{m}$ denotes the connected component of $V \backslash \tilde{K}_{0} \tilde{a}_{i j}^{m}$ is defined as

$\tilde{a}_{i j}^{m}=\tilde{a}_{i j}^{n}$ if $\tilde{\mathcal{C}}^{m} \cap \mathcal{C}^{n} \cap(V \backslash U) \neq \emptyset$ (there is at most one $n$ satisfying this condition),

$$
\tilde{a}_{i j}^{m}=\delta_{i j} \text { if } \forall n, \tilde{\mathcal{C}}^{m} \cap \mathcal{C}^{n} \cap(V \backslash U)=\emptyset .
$$

Remark 9.2. - If $\forall n, \tilde{\mathcal{C}}^{m} \cap \mathcal{C}^{n} \cap(V \backslash U)=\emptyset$ then $\tilde{\mathcal{C}}^{m} \subset U$ is bounded and one can lower the energy by taking $\tilde{u}$ constant in $\tilde{\mathcal{C}}^{m}$ whatever the definition of the definite positive matrix $\tilde{a}_{i j}^{m}$.

We will now concentrate on each connected component $\tilde{\mathcal{C}}^{n}$. There is an orthonormal basis of $\mathbf{R}^{3}$ in which matrix $a_{i j}^{n}$ is diagonal. We denote by $b_{i}^{n}$ the element of the diagonal in this basis and by $\left(U_{0}^{1}, U_{0}^{2}, U_{0}^{3}\right)$ the components of $u_{0}$ in the same basis. The energy of $(u, K)$ in a ball $B_{R}$ can be written as

$$
\int_{\mathcal{C}^{n} \cap B_{R}} \sum_{i, j} a_{i j}^{n} \nabla u_{0}^{i} \cdot \nabla u_{0}^{j}=\int_{\mathcal{C}^{n} \cap B_{R}} \sum_{i} b_{i}^{n}\left\|\nabla U^{i}\right\|^{2} .
$$

Obviously, since $\left(u_{0}, K_{0}\right)$ is a global-minimizer, $U_{0}^{i}$ is harmonic and satisfies Neumann boundary conditions on $\partial \mathcal{C}^{n}$. Therefore, if $\partial \mathcal{C}^{n}$ is connected, theorem 3.1 and remark 3.2 implies that the functions

$$
r \mapsto \frac{\int_{\mathcal{C}^{n} \cap B_{r}}\left\|\nabla U^{i}\right\|^{2}}{r}
$$

are nondecreasing functions of $r$. Therefore the analysis of section 4 gives the

THEOREM 9.3. - If $\left(u_{0}, K_{0}\right)$ is a global-minimizer of $E$ such that $K_{0}$ is connected then $\left(u_{0}, K_{0}\right)$ is one of the following:

(i) $K_{0}$ is empty and $u_{0}$ is constant. 
(ii) $K_{0}$ is a straight line defining two half-planes and $u_{0}$ is constant on each half-plane.

(iii) $K_{0}$ is the union of three half lines with angle $2 \pi / 3$ and $u_{0}$ is constant on each sector.

(iv) in a polar set of coordinates and in the appropriate basis of $\mathbf{R}^{3}$

$$
U^{i}(r, \theta)=\alpha_{i} \sqrt{\frac{2}{\pi}} \sqrt{r} \cos \frac{\theta}{2} \quad \text { for } \theta \in[0,2 \pi)
$$

and $K$ is the half axis $\theta=0$. The constants $\alpha_{i}$ are such that $\sum_{i} b_{i}\left(\alpha_{i}\right)^{2}=1$.

Consequently, it is easy to check that the proofs presented in sections 5 to 8 for a black-and-white image holds for the case of a color image. Theorems 1.1 to 1.5 are true for $(u, K)$ minimizer of the energy $E$ defined in equation (9.3)-(9.1) and under condition (9.2).

\section{APPENDIX}

Proof of proposition. 7.1. - Let $w$ be the solution of $\Delta w=u-g$ in $B_{4 R}^{+}$ with Dirichlet conditions $w=0$ on $\partial B_{4 R}^{+}$. Since $u$ and $g$ are in $L^{\infty}$ and $K$ is $C^{1, \alpha}$ then theorem 5.5.5' of [18] gives that $w$ is in $C^{1, \alpha}\left(B_{2 R}^{+}\right)$.

We would like now to prove that $v=u-w$ is also in $C^{1, \alpha}\left(B_{2 R}^{+}\right)$. By construction $v$ is harmonic, it has an adjoint $V$ such that $v+i V$ is holomorphic. We may assume that $V(P)=0$. If $\gamma$ is an oriented regular curve connecting two points $A$ and $B$ in the interior of $B_{2 R}^{+}$and if $\nu$ denotes the normal to $\gamma$ then

$$
V(B)-V(A)=\int_{\gamma} \frac{\partial v}{\partial \nu}=\int_{\gamma} \frac{\partial u}{\partial \nu}-\frac{\partial w}{\partial \nu}
$$

For any points $M$ of $K \cap B_{2 R}$ let $\mathcal{C}_{M}$ be the portion of $K$ connecting $P$ and $M$. Since $w$ is in $C^{1, \alpha}\left(B_{2 R}^{+}\right)$we may introduce a function:

$$
\chi(M)=\int_{\mathcal{C}_{M}} \frac{\partial w}{\partial \nu}
$$

For any point $(x, y)$ of $B_{2 R}(P)$ we may define $\chi$ by

$$
\chi(x, y)=\chi(x, f(x))
$$

The function $\chi$ is then $C^{1, \alpha}$ in $B_{2 R}(P)$. 
Assume that $A$ and $B$ are two points of $K$. The curve $\gamma$ connecting $A$ to $B$ can be chosen arbitrary close to $K$. Lemma 3.1 of [6] and equation (10.1) give then

$$
V(B)-V(A)=\chi(B)-\chi(A)
$$

Since we assumed $V(P)=0$, we have for all $M \in K \cap B_{2 R}$,

$$
V(M)=\chi(M)
$$

Since $\chi$ is $C^{1, \alpha}$, theorem 5.5.5' of [18] give now that $V \in C^{1, \alpha}\left(B_{R}^{+}\right)$. The adjoint $v$ of $V$ is in the same space and we have proved that $u \in C^{1, \alpha}\left(B_{R}^{+}\right)$ which completes the proof of proposition 7.1.

\section{ACKNOWLEDGEMENT}

I am very grateful to J.-M. Morel for many discussions and his interest in this work. I would like to thank him, F. Dibos and E. Séré for introducing me to this problem.

\section{REFERENCES}

[1] H. W. Alt, L. A. Caffarelli and A. Friedman, Variational problems with two Phases and their free boundary, Trans. Am. Math. Soc., Vol. 282, 1984, pp. 431-461.

[2] L. Ambrosio, Existence theory for a new class of variational problems, Arch. Rat. Mech. Anal., Vol. 111, 1990, pp. 291-322.

[3] L. Ambrosio and D. Pallara, Partial regularity of free discontinuity sets I, to appear.

[4] L. Ambrosio, N. Fusco and D. Pallara, Partial regularity of free discontinuity sets II, to appear.

[5] G. Congedo and I. Tamanini, On the existence of solutions to a problem in multidimensional segmentation, Ann. Inst. Henri Poincaré, Vol. 8, 2, 1991, pp. 175-195.

[6] G. Dal Maso, J.-M. Morel and S. Solimini, A variational method in image segmentation: existence and approximation results, Acta Matematica, Vol. 168, 1992, pp. 89-151.

[7] G. David and S. Semmes, On the singular sets of minimisers of the Mumford-Shah functional, to appear in J. Math. Pures Appl.

[8] G. David, $C^{1}$-arcs for minimisers of the Mumford-Shah functional, to appear.

[9] F. Dibos, Uniform rectifiability of image segmentations obtained by a variational method, J. Math. Pures and Appl., Vol. 73, 1994, pp. 389-412.

[10] F. Dibos and G. Koepfler, Color segmentation using a variational formulation, preprint CEREMADE.

[11] E. De Giorgi, M. Carriero and A. Leaci, Existence theorem for a minimum problem with free discontinuity set, Arch. Rat. Mech. Anal., Vol. 108, 1989, pp. 195-218.

[12] L. Evans and R. Gariepy, Measure theory and fine properties of functions, London: CRC Press, 1992. 
[13] K. J. Falconer, The geometry of fractal sets, Cambridge University Press, 1985.

[14] H. Federer, Geometric measure theory, Springer-Verlag, 1969.

[15] G. Hardy, J. E. Littlewood and G. Pólya, Inequalities Second Edition, Cambridge university Press.

[16] U. Massari and I. Tamanini, Regularity properties of optimal segmentations, Journ. reine angew. Math., Vol. 420, 1991, pp. 61-84.

[17] J.-M. Morel and S. Solimini, Variational methods in Image Segmentation, Birkhauser, 1994.

[18] C. B. Morrey Jr., Multiple integrals in the calculus of variations, Springer-Verlag, 1966.

[19] D. Mumford and J. Shah, Optimal approximations by piecewise smooth functions and associated variational problems, Comm. on Pure and Appl. Math., Vol. XLII, $\mathrm{n}^{\circ} 4$, 1989.

(Manuscript received;

Revised.) 